MODESTY DURING CHILDBIRTH:

PERSPECTIVES OF IMMIGRANT MUSLIM WOMEN IN CANADA

by

Sanjana N. Khan

Bachelor of Arts, Early Childhood Education,

Ryerson University, Toronto, ON, 2006

\author{
A Major Research Paper \\ presented to Ryerson University \\ in partial fulfillment of the requirements for the degree of \\ Master of Early Childhood Studies \\ in the program of \\ Early Childhood Studies
}

Toronto, Ontario, Canada, 2010

(C) Sanjana Khan 2010 


\section{Author's Declaration}

I hereby declare that I am the sole author of this major research paper. I authorize Ryerson University to lend this major research paper to other institutions or individuals for the purpose of scholarly research.

I further authorize Ryerson University to reproduce this major research paper by photocopying or by other means, in total or in part, at the request of other institutions or individuals for the purpose of scholarly research. 


\title{
Modesty during Childbirth: Perspectives of Immigrant Muslim Women in Canada
}

\author{
Sanjana N. Khan \\ Master of Arts, Early Childhood Studies \\ Graduate Studies \\ Ryerson University \\ 2010
}

\begin{abstract}
According to the Qur'an, modesty, the need to cover one's body, especially in the presence of members of the opposite sex, is an important principle for all Muslims.

Maintaining modesty becomes a significant issue, especially for immigrant Muslim women when they experience childbirth in a country, such as Canada, where health care professionals who specialize in obstetrics and gynecology can be both women and men. The purpose of this study was to understand Muslim women's ideas toward maintaining modesty in the specific context of childbirth. I used a qualitative inquiry approach to conduct interviews with four immigrant Muslim women who experienced childbirth at different hospitals in the Greater Toronto Area. The findings showed that while the views of participants towards modesty sometimes differed, their views toward modesty in the specific context of childbirth were similar, in that they all wanted to be cared for by exclusively female health care professionals and they all wanted to have their bodies covered as much as possible, for as long as possible (during their childbirth experiences) and as soon as possible (after delivery) to ensure minimal exposure. Recommendations are provided to improve care to Muslim women undergoing childbirth at hospitals in the Greater Toronto Area.
\end{abstract}




\section{ACKNOWLEDGEMENT}

First and foremost, I extend my sincere and heartfelt gratitude to my Creator for providing me with this opportunity and the ability to pursue and see this Major Research Paper (MRP) through to its fruition.

Since this is a study about women's experiences during childbirth, it is only fitting that I begin by acknowledging my amazing mother, Akhtarunnisa Khan. I thank her for enduring childbirth so that I could be born. She is my inspiration and I am grateful that she was chosen to be my mother.

I would also like to convey my genuine appreciation to my wonderful husband, Ruhul Amin Maula, who convinced me to pursue this MRP. His confidence in me and his unconditional support throughout this entire process gave me the motivation I needed to complete this endeavour.

Most especially, I would like to thank my supervisor, Dr. Sepali Guruge, for inspiring and supporting me throughout my journey and for investing countless hours of her time to guide, edit and proofread my writing. I truly appreciate her patience, dedication and commitment to direct my novice effort.

I would also like to thank Dr. Mandana Vahabi for accepting the role of second reader for this MRP, on such short notice.

My genuine appreciation is also offered to all of the wonderful women who agreed to participate in this study and trusted me with the personal stories of their childbirth experiences.

I would also like to acknowledge my good friend and peer, Nina Camargo Ramos for encouraging me throughout this process. 


\section{DEDICATION}

This MRP is dedicated to my Maryam Amina my Shona Bachcha, Manik Sachcha

and to the memory of my father,

Mohiuddin Ahmed Khan. 


\section{Table of Contents}

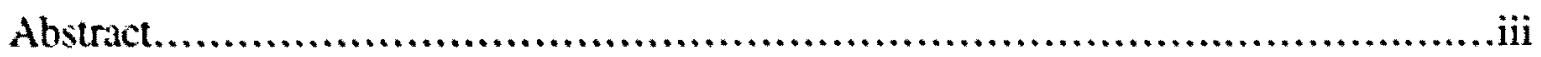

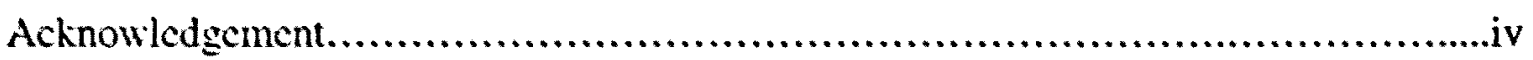

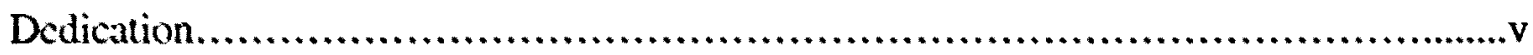

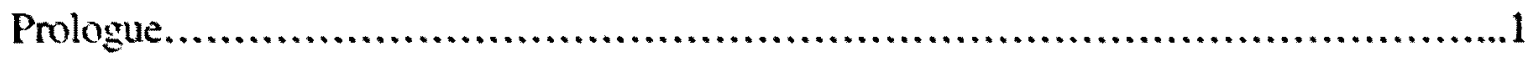

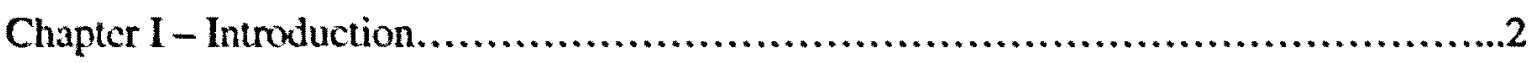

Chapter II - Literature Review.......................................................

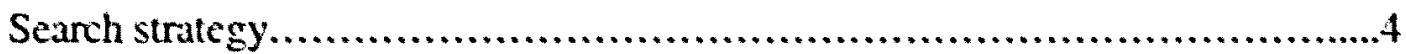

Key areas of the literature review.............................................4

Global perspectives on modesty during childbirth.........................4

Islamic perspectives on modesty during childbirth........................5

Immigrant women's perspectives on modesty during childbirth............6

Woman-centered care ...................................................

Rationale for the study and research focus ..................................10

Pre-study reflections..........................................................11

Theoretical perspectives......................................................11

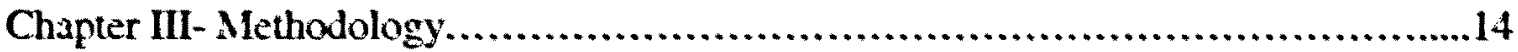

Qualitative inquiry........................................................14

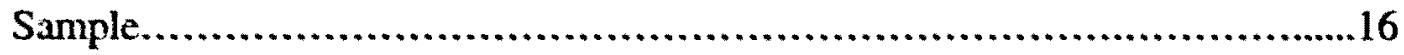

Risks and Benefits........................................................

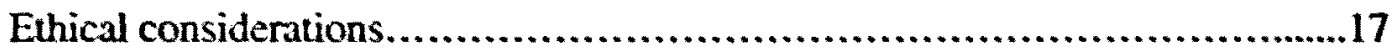

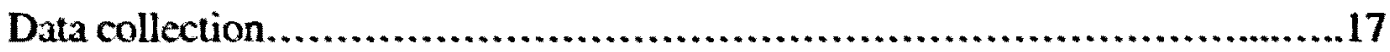

Data organization and analysis.................................................19 
Trustworthiness.

Chapter IV- Results.

Basic demographic information..............................................21

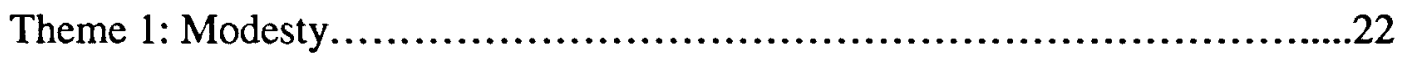

Theme 2: Women's Expectations..........................................27

Theme 3: Childbirth and care experiences...................................32

Theme 4: Recommendations....................................................45

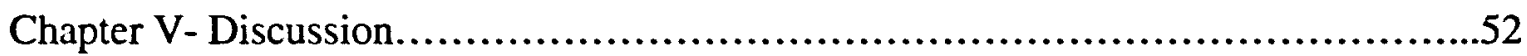

Study strengths and limitations.......................................64

Chapter VI- Recommendations................................................66

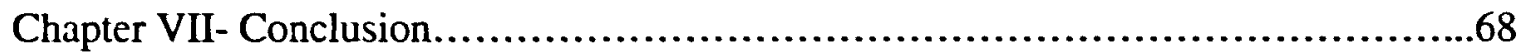

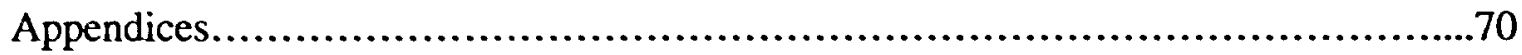

Appendix A: Information and consent agreement for participants.....................71

Appendix B: Interview guide questions for participants...............................74

Appendix C: Copy of my colour-coded working notes..................................76

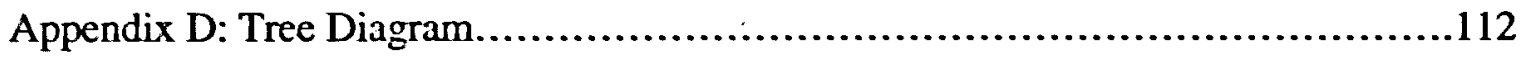

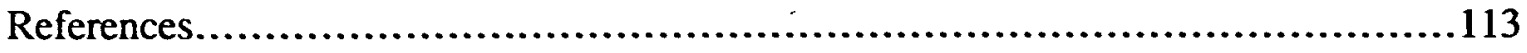




\section{PROLOGUE}

Before we ask others to furnish us with lived-experience description about a phenomenon...we might do well to try such descriptions ourselves first, so that we have a more precise sense of what we are attempting to obtain (Van Manen, 1990, p. 64).

The impetus for this study is a result of my own lived-experience of giving birth to my daughter at a hospital in Toronto, Canada, in 2006. I am a Muslim. I practice Islam, a religion in which modesty (need to keep body and hair covered) is highly regarded. Islam also places great importance on health and wellbeing. During the delivery of my daughter, my needs related to modesty were not regarded at a major hospital in Toronto. In particular, my request to not to be examined by a male student during my labour was ignored. I was informed that it would not be possible as the male student needed to have patients to 'practice on'. Helplessly I gave in and endured through an examination that left me humiliated and embarrassed. In retrospect, I realize that I did not fight hard enough to exercise my right because my ideas of modesty might have changed over time. This led me to want to learn about other Muslim women's ideas about maintaining modesty during childbirth. Although bound by religious parameters, Muslim women can have significantly differing views on the issue of modesty. 


\section{INTRODUCTION}

As one of the three major immigrant accepting cities in Canada, the population of the City of Toronto consists of many diverse individuals and groups. This diversity means having to tend to the varied needs of individuals as well as the specific cultural groups to which they belong. This Major Research Paper will focus on a group of immigrant Muslim women who have given birth in a hospital setting in the Greater Toronto Area. This topic is of significance because it addresses the often overlooked needs of Muslim women who prioritize modesty, especially in the context of childbirth where they feel most vulnerable. Such ignorance may have serious psychological consequences on the health and wellbeing of child-birthing women.

Although the word 'modesty' has various definitions, my focus will be on modesty in dress, speech, and conduct. One of the dictionary definitions of modesty that is offered by Ben-Ze-ev (1993) is, "having or showing regard for the decencies of behaviour, speech, dress, etc" (p. 235). This definition closely relates to Islam's perception of modesty as well. The Hadith of Sahih-al Bukhari is a respected Islamic text that contains the prophetic traditions of the Islamic Prophet Mohammed, which serve to better understand the meaning of the Qur'an. The Qur'an is the most important Islamic text that is accepted by Muslims to be the word of God. According to the Sahih al-Bukhari, modesty (haya) is a part of faith [and is] reflected in a Muslim's speech, dress and conduct. Maintaining modesty in dress, specifically in the presence of men (with the exception of their husband), becomes a significant issue in the context of childbirth, when women are most exposed and vulnerable. According to The Fatwa Center of the Islamweb website (1999), the fatwa (ruling) on a Muslim woman being examined by a male doctor is as follows: 
It is prohibited for a female to expose her Awrah (those parts of the body, which must be covered) to any man; she has to cover herself from him. If she needs to uncover her Awrah for any legal reason, such as illness etc., she can do that provided that she can not find a female doctor and that the disease is so serious that delaying the treatment may be dangerous for her life or for any part of her body. In these conditions or similar ones, she can expose her body to a male doctor and it should be in the presence of her husband or of any un-marriageable relatives. If they are not found, at least there should be a female nurse (Fatwa 82093, 1999).

As the above quote informs, although it is an expectation for Muslim women to maintain modesty in the presence of male health care professionals in the context of medical examinations, Islam allows exceptions to be made in cases of life-threatening emergencies. 


\section{LITERATURE REVIEW}

\section{Search strategy}

The following review is based on literature found via the CINAHL, EBSCO and Proquest databases. Key words used were: Modesty, Muslim Women, Childbirth, Culture, Ethnic, Immigrant, Islam, and Religion. Although most of the review is comprised of literature published between 1999 and 2009, there are also a select few references to particularly relevant literature published between 1991 and 1998. All of the literature reviewed were published in English, and based on research conducted in Canada, the United States, Zambia, Somalia, Turkey, England and Australia.

The following is a summary of the literature, presented under four key areas: 1) global perspectives on modesty during childbirth; 2) Islamic perspectives on modesty during childbirth; 3) immigrant women's perspectives on modesty during childbirth; and 4) woman-centered care.

\section{Global perspectives on modesty during childbirth}

Khalaf and Callister (1997) describe childbirth as a culturally and spiritually significant event in a woman's life. Roberts (2002) corroborates with that, "the birth experience represents a time of great reflection and intimacy for individuals of all religions" (p. 32). Arif (2006) affirms the multi-spiritual belief in modesty stating, "Major religions- Hinduism, Islam, Buddhism, Sikhism and Christianity exhort their followers to adopt some aspect of modesty, such as covering the body or head and limiting social interaction with the opposite sex" (p. 32). Arif refers to the following anecdote shared by a female patient (religion unspecified) to illustrate that the significance of modesty and how in this situation, the patient's concern for modesty went unnoticed: 
I was experiencing chest pains and was admitted to hospital as an emergency. I was treated like a piece of meat. They gave me a hospital gown that was supposed to tie at the back, but there were no ties. When a nurse started attaching electrodes to my body with scant attention to my comfort or exposure, my daughter quickly drew the curtains around my bed. A male nurse barged in. Never mind my pain; I was so ashamed to be seen like that. Why could he not give me a moment so I could cover myself? I could not use the bathroom with my back uncovered. Bedpans made me feel dirty and useless. I should have just died at home (p.33).

Arif (2006) further extends this acknowledgement to establish the fact that modesty plays a significant role when it comes to providing culturally and spiritually sensitive care. A study conducted in Zambia by Maimbolwa, Yamba, Diwan and Ransjo-Arvidson (2003) explored the process of childbirth from the perspective of Mbusas (women who implement traditional reproductive practices), and found that the preferred method of childbirth was to deliver at home, with the assistance of midwives, whereby eliminating the possibility of having the child-birthing women's modesty breached in the presence of men. De Sevo (1997) notes the importance of maintaining modesty in childbirth in Jewish traditions as well, one of which happens to be the need to have their heads covered. The notion of "maintaining the integrity of the client during high-stress experiences" was highlighted.

\section{Islamic perspectives on modesty during childbirth}

Arif (2006) affirms that, "In Islam, the [Qur' an] further urge[s] all Muslims [men and women] 'to cast their eyes down' in mixed company. In other words, to show respect and maintain modesty in thought and deed" (p. 32). For Muslim women who abide by the way of the Qur'an, the inability to control for male presence in the labour-room is of highest concern, and can result in a negative and stressful experience. Islam advocates modesty in all non-emergency situations, but is also open to accepting and accommodating for emergency circumstances. Islam's understanding of the need to do whatever is necessary 
during emergency situations is evident in Qur' an [albeit on a different, but equally important issue], which states,

He [Allah] has only forbidden you what dies of itself, and blood, and [the] flesh of swine, and that over which any other [name] than [that of] Allah has been invoked; but whosoever is driven to necessity, not desiring, nor exceeding the limit, no sin shall be upon him; surely Allah is Forgiving, Merciful." (Chapter 2, Verse 173).

This reasoning of being driven to necessity can be applied in the child-birthing context as well to justify the presence of male medical professionals during the child-birthing process, and in emergency situations, when no other option (i.e. other qualified female practitioners) is available.

Yanikkerem, Ozdemir, Bingol and Karadeniz (2009) conducted a study in Turkey, a predominantly Islamic country, and found that of the 433 women participants, a significant majority ( $45.5 \%)$ reported that they would prefer a female doctor, while only $4.2 \%$ preferred a male doctor. The authors further found that the latter was based on the socially promoted belief that men make better doctors. Shorten (1995) found that Somali women (predominantly Muslim) also believe in the preservation of modesty and consider privacy to be crucially important, especially in childbirth related situations.

\section{Immigrant women's perspectives on modesty during childbirth}

Historically, childbirth in the west, similar to non-western countries, was attended by midwives, in the home and with support from other women in the family and in the community (Condon, 2004). This author further states that "men generally did not witness births and male physicians [were] scarce and did not begin to supplant midwives until the late 1700 s" (p. 6). Hospitalization was only required in cases of emergency. Condon further informs that the "prohibition against the display of the female body was so strong 
that medical education [which was exclusive to men] did not include the direct observation of childbirth" (p. 6). Maintaining modesty during childbirth, thus, was not an issue until the frequency of childbirths attended to by male physicians increased. Since the advent of institutionalized childbirth, the potential for issues of modesty to be breached has become a significant issue. Fenwick, Hauck, Downie and Butt (2005) note, "As childbirth becomes an increasingly medicalised event, characterised by technology and spiraling intervention rates, it is important to explore current childbirth expectations in the hope that maternity systems and health care professionals can better meet women's individual needs and preferences" (p.33).

According to The College of Physicians and Surgeons of Ontario (CPSO) website, as of August 7, 2010, 206 doctors specialize in obstetrics and gynecology in Toronto. Of these, 100 (48.5 percent) are women. This number suggests that it should be fairly easy to have access to care by a female OB/GYN (Obstetrician/Gynecologist). Statistics Canada reports that according to the 2006 Census, the City of Toronto received the highest number of immigrants in Canada. Immigrant women who give birth at Toronto hospitals may come from villages in countries, such as Bangladesh, India, and China, that may still be reliant on the home-delivery via midwife protocol. It is also important to note that in recent years, the preference for midwives has increased in Canada as well. In 2006, The Maternity Experiences Survey (an initiative of the Canadian Perinatal Surveillance System of the Public Health Agency of Canada in conjunction with Statistics Canada) with 6,421 mothers (citizenship status unspecified) showed that the women [were] happier with births overseen by midwives, with 71 per cent of women who had a midwife delivery rating it as 'very positive'"' (II 6). 
In an award-winning article, Young (2002) "[explores] issues pertaining to women who have given birth in a foreign country... and [seeks to understand] the relevance [of these issues] to nurses and midwives through the implementation of culturally competent care" (p. 16). Young cites a number of examples of groups of women who for various cultural and religious reasons do not wish to expose their genital area to anyone other than their husbands. Chalmers and Hashi's (2002) phenomenological study with 432 Somali women who had given birth in Canada found that the women were dissatisfied with the care they received as they felt that their caregivers lacked knowledge of their cultural practices. Reitmanova and Gustafson's (2008) Canadian study deals specifically with the maternity health and care needs of immigrant Muslim women in St. John's, Newfoundland. All of the participants in the study stated a strong preference for having a female attendant care for them during their childbirth experience, and agreed that having a male physician, although uncomfortable, was acceptable only if there was no other options.

\section{Woman-centered care}

Sperstad and Werner (2005) believe that although it may be helpful to begin with generalizations to understand potential cultural beliefs and practices, it is important to be cautious about stereotyping. No matter what culture we identify with, who we are, depends on the way we have experienced events, people, faith and numerous other factors that shape our individual existence. Leap (2009) supports this need to have 'woman-centered' versus 'women-centered' care, where the locus of control shifts away from the institution and professionals to the woman herself. What is appropriate for one woman may not be for another. The importance of the care that results from an individualized needs assessment is 
further highlighted in the philosophy statement of The Australian College of Midwives

(2010) that defines 'yoman-centered care' as:

- Holistic in its approach and recognizes each woman's social, emotional, physical, spiritual and cultural needs, expectations and context, as defined by the woman herself.

- Recognizant of every woman's right to self-determination in attaining choice, control and continuity of care from one or more known caregivers.

- Encompassing the needs of the woman's baby, and includes the woman's social, emotional, physical, spiritual and cultural needs, expectations and context as defined by the woman herself.

- Recognizant of every woman's responsibility to make informed decisions for herself, her baby and her family with assistance, when requested, from health professionals (II 9).

The rise in women opting for home-births with the help of midwives supports the assumption that increasing numbers of women are attracted to the facets of midwifery care as stated above. An article in Nursing (2006) refers to the National Center for Health Statistics (2003) that in the U.S. alone, the number of women giving birth with the assistance of a midwife has more than doubled since 1990 .

Fenwick, Hauck, Downie and Butt (2005) explored child-birth expectations of Australian women and found that individual women's beliefs and expectations regarding childbirth differed significantly from one another. This finding supports the philosophy statement of the Australian College of Midwives (2010) that there is a need for 'womancentered' care.

Young (2002) refers to the United Kingdom Code of Professional Conduct (1993), which requires health care professionals to "recognize and respect the uniqueness and dignity of each patient and client and respond to their need for care irrespective of their ethnic origin, religious belief, personal attributes, the nature of their health, or any other factor" (p.18). This notion of individualized care is crucial because, as Simkin (1991) 
states, the memories women hold [especially] of their first childbirth experiences has an immense psychological effect on their lives and that the memories depend greatly on the amount of control they had. Simkin further asserts,

A woman in labour is highly vulnerable. Her most private body parts are exposed; she is in pain; she sweats, trembles, moans, and cries out while among strangers; she is in a strange environment. If she is treated without respect, if her efforts to maintain dignity and control are rebuffed, or if she is taken advantage of, the negative impact is permanent. If she is nurtured, treated with kindness and respect, and feels like a participant, the positive impact is permanent (p. 210).

In summary, this review of literature shows that while much has been written about the need for culturally sensitive care in hospitals and the experiences of Muslim women in hospitals, the research on the specific issue of the need for Muslim women to maintain their modesty during pregnancy, labour and childbirth (when women are most vulnerable as they require the most intimate parts of their body to be exposed) is limited.

\section{Rationale for the study and research focus}

Prior to determining my topic for this paper, I engaged in informal conversations about child-birthing experiences with several Muslim women. The single-common thread that linked all of our stories was the fact that we all felt uncomfortable and voiceless at a time when we were most vulnerable. It is through these informal conversations that I realized that women's (in this case, Muslim women's) voices need to be heard and their sentiments understood. As such, this study was conducted to answer to the following overarching question: What are Muslim women's views towards, and experiences of, maintaining modesty during childbirth? 


\section{Pre-study reflections}

Prior to engaging in the actual study itself, I reflected on what I might expect to find and what my thoughts are about the subject under investigation. The following is a list of my biases and reflections about the topic.

1) I predicted that most of the women would view modesty, especially modesty in dress, as a positive facet of their faith, something that protects them from receiving unwanted attention.

2) I expected that most of the women would further state that their modesty in dress enables them to feel empowered. When they speak, people listen and focus on what they say and are not distracted by their physical appearance.

3) Moving onto the issue of maintaining modesty during childbirth, I expected that all the women would have strong opinions regarding this matter. I expected that the experiences of those who wear and do not wear hijab would be different because health care professionals may be able to predict or anticipate the modesty needs of those who wear the hijab. In other words, I expected that the inability to identify a Muslim woman may also emerge as a theme.

4) As with my case, I expected that women's request to only be examined by female nurses or doctors in training may be disregarded, leaving the participants feeling helpless, humiliated, and rejected.

\section{Theoretical perspective}

I used a combination of two frameworks to guide my study: the cultural competence approach and the intersecting identities frameworks. 


\section{Cultural competence approach}

This approach is rooted in transcultural nursing, which was first introduced in the 1960 s by Madeleine Leininger (Gustafson, 2008). Culture, in this context, is defined as a shared set of values, behaviours and beliefs that is "held by a group that shares common attributes such as race, language or religious affiliation" (Gustafson, 2008, p. 43). This approach advocates for health care professionals to "become aware of her or his culture, the client's culture and any similarities or differences between cultures that may negatively impact on the client's health and health practices, and the therapeutic relationship between client and practitioner" (Gustafson, 2008, p. 43).

My reasoning for using this framework is based on both the definition of culture, as well as the focus on the client-practitioner relationship, as mentioned above. The participants of my study share a belief in a common religion, Islam, and one of the primary objectives of this study is to determine how the cultural diversity of the clients (childbirthing women) were understood and addressed by the practitioners (OB/GYNs, nurses and midwives) in the context of childbirth. However, some of the practitioners of the cultural competence approach might not take into account the individual differences of the women. As such, I felt the need to incorporate the intersecting identities framework.

\section{Intersecting identities framework}

Guruge and Khanlou (2004) provide insight for the creation of new ways of inquiry that is necessary to explore and understand the way in which many dimensions of an individual's social identity intersect to influence the health care needs of immigrant women. Khanlou (2003) states that this concept of "intersectionalities of influence" occurs 
when various influences converge in unique ways, which in turn leads to individuals and groups experiencing different health outcomes.

Guruge and Collins (2008) further clarify that the intersecting identities framework is based on the belief that women's experiences are shaped by: A range of identities [that] intersect and change in various social contexts. As such, it is critical for [health care professionals] to consider women's unique experiences, rather than focusing on commonalities based on [only one aspect of their collective experience]" (p. 23). This framework is also appropriate for my study because although the study participants may share the same religion, the way in which they have each experienced and understood various aspects of life, including their common religion might be unique and may have been shaped by various social contexts. 


\section{METHODOLOGY}

A qualitative approach was used for this study. Denzin and Lincoln (2005) define qualitative research as "a situated activity that locates the participants, researchers and observers in the world" (p.417) and qualitative researchers as those who study specific phenomenon in natural settings to understand why things happen from the point of view of the people experiencing it. Creswell (2007) identifies several reasons for conducting qualitative research, including:

a) to empower individuals to "share their stories, hear their voices and minimize the power relationships that often exist between researcher and participants"; b) to understand the contexts (home, family, etc.) in which participants address an issue, because we cannot separate what people say in relation to the context in which they say it;

c) to gain a detailed understanding of the issue by giving participants the opportunity to tell their stories, without being restricted by what we expect to find based on what we have read in literature;

d) to investigate how a specific phenomenon affects an individual, instead of how it applies to general population (p. 40).

My decision to use a qualitative approach for my study is to gain a detailed understanding of how Muslim women define modesty and the role it plays in the context of childbirth. Childbirth is experienced on an individual basis and as such needs to be addressed on an individual level. Also, there is limited literature available on the subject, warranting further and in-depth understand of the topic. Furthermore, in accordance with Creswell (2007), my study began with a single and specific focus (Muslim women's ideas regarding 
modesty during childbirth) and the nature of my question is such that it cannot be answered using the quantitative approach or statistical analyses. A qualitative approach is the best "fit" (p. 40).

\section{Sample}

The participants of this study consist of immigrant Muslim women who: 1) have had at least one childbirth experience in hospitals within the Greater Toronto Area within the last 10 years; 2) are older that 18 years of age; and 3 ) are able to use verbal and written communication in English to understand the intent of the study and provide consent to participate in the study. No organizations or institutions were involved in choosing participants. A convenience sample of my personal acquaintances who met the selection criteria specified above were invited to participate. Given the time limitations for my study, a sample of 4 participants is included.

During the initial recruitment process, assessing and maintaining a good rapport with the participants is essential in order to establish a trusting relationship. As a member of the Muslim community, I have known the 4 women who participated in this study for more than five years. Prior to presenting the consent form, I informed them of my research purpose in full, including any potential benefits and risks (as outlined in the following section), so that each participant had adequate information in order to make a free choice to participate or withdraw from the study. The participants were further made aware (in verbal and written form) about their right to refuse participation at any time and leave the study or ask me any questions they may have had regarding the study. All of the women received an information sheet and a consent agreement form (see Appendix A) a week prior to the actual interview session. This allowed them time for thought before deciding 
whether or not they wanted to participate in the study. To ensure that the participants did not feel coerced to participate because of our existing friendship, I included a statement in the consent agreement form that clearly stated that the decision to participate in the study (or not) was completely up to them and would not have any negative effects on their existing friendship with me or on any future interactions they may have with Ryerson University.

\section{Risks and benefits}

Potential risks included the participants' feeling apprehensive about the interview process, because they may not have engaged in such an activity before. They may have also had to consider and (in some instances) ask their husband's permission to participate. They may have also had concerns about arranging child-care for the duration of the interview.

To manage these risks, I ensured that the participants were informed about the details of the research project (in verbal and written form). One woman who had agreed to participate had to withdraw later due to certain unforeseen circumstances. I thanked her for considering my invitation and approached another individual from my list.

In situations where securing child care was an issue, I offered to make myself available at a time when their children were either at school or asleep. To ensure the comfort of all participants, I conducted the interviews personally.

Also, because the interviews revealed moments of the participants' lives that may have lead to emotional distress, I used the following strategies to manage the situation:

1) To minimize the potential for emotional discomfort, participants were informed of their right to refuse to answer any questions that they felt uncomfortable with. 
2) Participants were also given my contact information, including phone number and e-mail address in case they wanted to make changes to or extract any part of the text, prior to the end of the data collection period.

3) All interviews were conducted in a location that was safe, convenient and mutually agreed upon by the individual participant and the researcher.

Some of the (anticipated) benefits to participants included: 1) having their voices heard (including concerns, if any arise); 2) having their child-birth experiences matter; and 3) the possibility of having their experiences inform current health care practices in order to better support the needs of the Muslim women during childbirth.

\section{Ethical considerations}

The following measures were taken to ensure that confidentiality was maintained: 1) assigning numbers to participants from the onset of the study, so that the data would remain anonymous; 2) storing the consent forms and identifiers in a locked cabinet in my supervisor's office at Ryerson University and 3) storing the interview tapes in a separate locked cabinet in my supervisor's office at Ryerson University. None of the identifiers or raw data were available to anyone aside from me and my research supervisor.

\section{Data collection}

I used semi-structured, in-person, audio-taped interviews. As outlined by Creswell (2009), the individual interviews are useful: 1) when participants cannot be directly observed; 2) because they allow participants to provide historical information [and information about their lived experiences from her/his perspective]; and 3) because the interviews allow the researcher to have control over the line of questioning. 
I also took some notes during the interviews. This was limited to specific information that I felt required clarification, to be asked after the participant finished responding to a specific question. I also took notes about facial expressions and hand gestures, including smiles, grimaces, and pointing as they happened, because those could not be captured on audio-recording. These notes were used as reference to provide support for the use of words or phrases which needed visual aids to understand. For example, some of the participants pointed toward the general direction of their lower abdomen and legs as they used terms like "those parts". This helped me to confirm that the participants were speaking specifically about their legs and vaginal areas, as opposed to other parts of their bodies. One of the participants also grimaced when speaking of her childbirth experience in Hong Kong. Initially, I was confused because the participant did not clarify that the health care system in Hong Kong worked differently from the system in Canada. The participant was visibly upset when she spoke of her prenatal examinations. Her grimaces served as my cue to investigate the issue further. I then realized that the reason for her discomfort in recalling the memory of her first childbirth was directed toward the fact that her prenatal examinations were done by different doctors, instead of just one.

Following ethics approval from Ryerson University's Research Ethics Board, I began my interviews. In general, interviews lasted for approximately one hour in duration. Questions to participants focused on issues of modesty in general; modesty in the childbirthing context; and their experiences of maintaining modesty during their childbirth experience. See Appendix B for the list of interview questions. Some questions were modified during the process of the interviews, based on previously answered questions. The interview questions were open-ended to allow participants to share their thoughts 
without restrictions. The need for a second or follow-up interview did not arise. All interviews were done in English. Two audio-recording devices were used simultaneously, so that if one failed, the other would act as back-up. I stopped my data collection after I managed to extract complete and thorough answers to as many questions as possible from each of the women.

\section{Data organization and analysis}

In order to keep my data organized, I designated four file-folders (one for each participant) to contain information generated from individual participants. Each folder was labeled with a number to ensure that the participants' identities were protected. Each folder was then categorized into sections that included hand-written interview notes (taken by myself as a supplement to the audio-recordings) and actual interview transcripts.

I followed the guideless outlined by Creswell (2009) to conduct my data analysis and to validate accuracy of the data. I began by ensuring that all my data were organized (as described in the previous section) and by double-checking that all of the tapes were accurately transcribed. I then read through all the data to gain a general sense of the information and to try to extract emerging themes. To keep track of my thoughts, I wrote colour-coded notes in the margins. Next, I began coding using the eight-step process outlined by Tesch (1990). I relied on the actual words used by the participant to draw out the codes (ie., in vivo codes) (Creswell, 2009). I then arranged the codes under subheadings as suggested by Creswell (2009, pp.186-187). These headings were: 1$)$ expected codes; 2) unexpected codes; 3 ) codes that are unusual, and 4) codes that address a larger theoretical perspective. From there I used the larger codes to extract smaller themes, interconnect themes and describe themes as they emerged. (A copy of my working notes is 
provided in Appendix C). From these notes I worked with my supervisor to finalize the sub-themes and themes, and to generate a tree diagram (see Appendix D) to act as a visual aid to organize the themes and sub-themes.

\section{Trustworthiness}

Based on Creswell and Miller (2009), I undertook the following steps to ensure trustworthiness:

1) I used member checking. I took my coded transcripts back to the participants so they could review them and inform me if they felt that the themes I extracted were accurate.

2) Because the idea for the study was a product of my personal experience, I clarified the biases that I brought to the study at the beginning of the study (see Chapter 2) and kept these in my mind during interviews with participants, as well as during my discussions of data with my supervisor. The latter, in particular, helped me to question my interpretation of some of the findings and write-up of the findings.

3) I also reported any negative or discrepant information that came out as I reviewed emerging themes. This is important because such evidence indicate that the research is thorough and does not leave any information unused. 


\section{RESULTS}

\section{Basic Demographic Information}

The four participants are in their early to late 30 s, and all of their childbirth experiences occurred while they were in their 20s. Two of the women are from Bangladesh, one is from Pakistan, and another from Hong Kong. Their professions are varied. One is a doctor, one a nursing student, one is a childcare provider, and one is a stay-at-home mother. The participants also vary in the type of modest attire they choose to wear. One woman wears a hijab (scarf mainly meant to cover the hair) and an abaya (long, loose-fitting Islamic dress, meant to cover the whole body), another woman wears a hijab with loose-fitting Western outfits that cover the whole body, and the final two women alternate between loose-fitting Western and cultural clothing without hijab.

Each participant had experienced at least one childbirth occurrence in a hospital in the Greater Toronto Area. Two women had two childbirth experiences; one had one, and one had three. Two participants had their first childbirth experiences in hospitals outside of Canada (one in Hong Kong and the other in the United States). Three of the participants had their first Canadian childbirth experience within the first three years of immigrating to Canada. A summary of their demographic information is provided in Table I.

Table I: Basic Demographic Information of Participants

\begin{tabular}{|c|c|l|l|c|c|c|}
\hline \multirow{2}{*}{ Participant } & \multirow{2}{*}{ Age } & \multirow{2}{*}{$\begin{array}{c}\text { Country of } \\
\text { Origin }\end{array}$} & \multicolumn{1}{|c|}{ Profession } & \multirow{2}{*}{$\begin{array}{c}\text { Years in } \\
\text { Canada }\end{array}$} & \multicolumn{2}{|c|}{ Number of Children Born } \\
\cline { 5 - 7 } & & & Outside Canada & In Canada \\
\hline $\mathbf{1}$ & 39 & Bangladesh & $\begin{array}{l}\text { Child Care } \\
\text { Provider }\end{array}$ & 25 & 0 & 2 \\
\hline $\mathbf{2}$ & 32 & Hong Kong & Nursing Student & 10 & $1^{*}$ & 2 \\
\hline $\mathbf{3}$ & 35 & Pakistan & Doctor & 15 & 0 & 1 \\
\hline & 38 & Bangladesh & $\begin{array}{l}\text { Stay-at-Home } \\
\text { Mother }\end{array}$ & 10 & $1^{* *}$ & 1 \\
\hline
\end{tabular}

* Born in U.S., ** Born in Hong Kong 
Based on the analysis of data collected, four themes were generated. They are: modesty, expectations, childbirth experiences, and recommendations. Each theme is related to several sub-themes, which are discussed in detail in the following sections.

\section{Theme 1: Modesty}

Modesty can have different meanings based on cultural, social and religious contexts. Based on the interviews, the findings presented here are limited to the participants' understanding of the Islamic views of modesty which are embedded within their personal perceptions of modesty as well. More specifically, modesty as a means of covering the body (dress) and guarding one's demeanor (behaviour and speech) within the specific context of childbirth is examined.

\section{Islamic and personal views of modesty}

As most of the participants pointed out, modesty, according to Islam is reflected in the way one lives one's life and is evident in not only their dress, but in their behaviour and speech as well. The following are some examples of each participant's understanding of modesty, some from a religious point of view and some from a personal perspective:

Islam describes modesty... when... you should dress properly, so people, you know, would have respect for you when they see you. (Participant 4, Age 38).

Hair is a symbol of beauty for women and you're supposed to have it covered up so that no other man will be interested in looking at you. So, according to the religion, you know, that's why women supposed to be covered. (Participant 2, Age 32).

I think for Muslim women, modesty means that you have to be covered up, exposing only your face and your hands and...you are not supposed to show any other parts of your body in front of other people, especially other men. And no other man should have any physical contact with you, or see any part of your body. (Participant 2, Age 32).

Islam teaches that we should apply this kind of behaviour by speaking softly and politely with others. (Participant 4, age 38). 
I remember one time I was in the mosque and there was a little bookstore...I was looking at one of the books and it said, the fragrance of paradise (heaven) won't reach a woman for 500 years if she doesn't wear a head scarf. That's when I realized how important that is. (Participant 2, Age 32).

[Modesty] represents balance. Coming across as a Muslim woman, educated, living in a civilized society, who is not oppressed, who knows her rights, who knows how to carry herself in public and the way she dresses, although represents the fact that she is a Muslim, does not necessarily make her, um...stand out in a crowd, neither does it make her disappear. (Participant 3, Age 35).

I think modesty is not just the way you dress, it's an attitude as well. (Participant 2, Age 32).

The above quotations illustrate that, with the exception of dress, the participants' personal interpretations of modesty were in agreement and adhered to the basic principles of modesty as outlined by Islamic etiquette, as much as possible. The following section explores the notions of modesty in dress and speech as necessary components of the larger sub-theme of modesty in behaviour.

Modesty in behaviour

Modesty in behaviour was perceived by all of the participants to be an important aspect of their day-to-day existence. One of the women described modesty in terms of 'covering-up' the hair as well as the body, but also in terms of speaking properly to others.

[Modesty] mean to me... how I feel about covering myself, body and including my personality...how I am showing off to the people lhow she presents herself in front of people] and how I am talking to people... has to have a limit and to keep that limit bound is modesty for me. (Participant 1, Age 39).

Other participants also shared similar sentiments:

Modesty should be in everything, like of course in dress, your [behaviour], you know, kind toward other people. (Participant 4, Age, 38).

I'm not just modest in the way I dress, I like to be modest in the way I [behave], in the way I live my life... in the way I interact with my patients, I try to come across as being professional and modest. (Participant 3, Age 35). 
One woman perceived modesty in behaviour as a way of presenting herself to others, and another participant saw modesty in behaviour as being an important aspect of the professionalism she has to demonstrate as a doctor. All of the participants believed in maintaining modesty in their behaviour and the image that they portrayed in the presence of others. This behaviour was reflected in their dress and speech as well, both of which are further explored below.

Modesty in dress. For all of the participants, dress was a significant part of the modesty they exhibited. However, this was the only sub-theme in which the women offered differing perspectives. Their personal preference in dress was evident not only from their comments, but also from the attire they chose to wear during the interview. This difference of opinion is reflected in the following excerpts:

According to the religion (Islam), it is something that you're supposed to do, and it is expected. But, no one has ever forced it on me. It's something that I chose to do, because that's part of the modesty for women. (Participant 2, Age 32).

I used to wear a hijab before I got married, and my husband didn't like it, so I quit it. The clothing I prefer is not really fit-fit, that's gonna show my body. Because most of the time we prefer to wear our cultural clothes... and I try my best to make those as...pretty loose. (Participant 1, Age 39).

In terms of modesty, your dress should be such that you are not exposing parts of your body that are unacceptable in public, which I think is general rules everywhere, in every society. I think it's a personal choice and although I myself don't feel like I am at a place where I would commit totally to hijab, but eventually, when I come to that place, I would be totally comfortable wearing hijab. (Participant 3, Age 35).

I think modesty is... you know...dress appropriately, look neat and tidy, and of course when a person sees you, he feels respect. [Appropriately] means cover your body. Hijab...or you wear the abaya... people can cover in different ways.

(Participant 4, Age, 38).

Although their understanding of modesty in dress, as expected in Islam, were similar, their personal interpretations varied greatly. For two of the women, covering the hair, as well as 
the body, was very important, so they wore hijabs. However, for the two other women, covering the body was more important than covering their hair. One of the latter participants changed her view of modesty in dress, after marriage, in order to accommodate her husband's preference. She used to wear a hijab before marriage and decided to take it off after marriage. She felt that making this switch was something she could compromise about. Nevertheless, she noted that if she had felt strongly about wearing the hijab, she would not have allowed her husband's preference to influence her to take it off. The wearing of a hijab is, thus, seen as a choice, something that is recommended in Islam, but not mandatory.

Modesty in speech. Modesty in speech was also addressed by all of the participants as being another significant aspect of modesty in behaviour. However, the examples they provided were very general and not explicitly spoken off by any of the participants. However, upon in-depth examination of the interviews, I noted that three of the participants inadvertently provided specific examples of how modesty in speech is engrained in their consciousness. These participants consistently used the term "private parts" or "those parts", and deliberately avoided using the words "vagina" or "vaginal" in their interviews. For example, one participant stated, "In a regular situation, it has to be a female (health care professional), because no man should look at, you know, any part of your body, you know like..." (Participant 2, Age 32). She did not complete the sentence because doing so would require her to use specific words, which she was not comfortable to do.

Another participant used the term "vaginal infection" on two occasions during the interview, but it was said with some hesitation. In every other instance, she used phrases 
such as "personal body parts" (Participant 4, Age 38). I further noted that I too was doing the same, without intending to. The only participant who used "correct" wording throughout the interview was the doctor. For example, on one occasion, while explaining the stages of labour she stated, "...first stage is when the cervix dilates, until the baby's head is basically crowned" (Participant 3, Age 35). Modesty in speech can, thus, also be considered to be an implicit and underlying practice that may be influenced by cultural and/or religious factors.

\section{Modesty during childbirth}

When it comes to the specific topic of childbirth, all of the participants were in complete agreement about wanting minimal exposure of their bodies, in general, and zero exposure of their bodies to male health care professionals. The following comments illustrate this agreement:

And I remember, um... in the more later stage of labour, there was this woman who came in with a...young man, and she said, "This is a nursing student, would you mind if he observes the childbirth?" And I said, "I don't mind students as long as it's a female". (Participant 2, Age 32).

My personal belief is that...I have to cover myself from any male person. I have to go to the doctor and I have to show them (referring to exposure), but it has to secure my personality (personal choice) that there shouldn't be any men around. (Participant 1, Age 39).

They (nurses) kept me covered as much as possible, as long as possible, which was my request...again, comes back to the vulnerability aspect, where you know you feel vulnerable and exposed. (Participant 3, Age 35)

If she is a woman doctor, it's fine with me, but if [he's] a male doctor it wasn't fine with me. (Participant 4, Age 38).

The above quotes illustrate the emphasis that each participant placed on their need to be "covered" and shielded from the presence of any male health care professionals. Two of 
the comments were stated in the past tense indicating that these participants were referring to their lived experiences, whereas the two other women used the present tense to connote their current preferences. All of the women presented this need to cover as a personal choice, without referring to the religious context.

\section{Theme 2: Expectations}

This section focuses on the pre-childbirth expectations that the women had toward the doctors, nurses and hospitals, in general. These expectations varied because the women are from different social, cultural and ethnic backgrounds. Two women are from Bangladesh, one from Hong Kong and one from Pakistan. One of the participants from Bangladesh, as well as the participant from Pakistan directly immigrated to Canada, and they had their first childbirth experiences here. The other Bangladeshi participant had lived in Hong Kong for several years before immigrating to Canada. Similarly, the participant who was originally from Hong Kong had first lived in the United States before moving to Canada. The latter two women had experienced their first childbirth in countries outside of Canada, so they spoke the differences that they noted. The same was true for another participant who, as a medical student, had observed other women's childbirth experiences in a country outside of Canada. Three of the participants also experienced childbirth, in Canada, within the first three years post immigration. In contrast, only one woman had been in Canada for over 20 years and had both her childbirth experiences here. Given these differences, it could be anticipated that the expectations of each woman would be different as well. These expectations are explored in the following sections and are divided into the 
following sub-headings: women's expectations of doctors; women's expectations of nurses; and women's expectations of hospitals.

\section{Women's expectations of doctors}

All of the participants had specific expectations of their doctors, particularly in regards to having their needs for modesty respected and met. Some of the women made it a point to speak about their expectations from the onset of their pre-natal care, whereas others did not. The woman who was not a recent immigrant informed her family doctor at the beginning of her first pregnancy that she would prefer a female (OB/GYN) to see her through from beginning to end. As a result, this participant had been referred to a hospital that has a high ratio of female clients to female health care professionals. The following is a retelling of her expectations to her family doctor:

First thing I approached to her (family doctor) that yes for my delivery and for my check-up during my pregnancy, I prefer a female doctor. If she (her family doctor) is not available, I prefer another female doctor. (Participant 1, Age 39).

As the above quote illustrates, for this participant, finding a female OB/GYN was a primary concern.

The two women, who had previously given birth outside of Canada, expected their doctors to be sensitive and accommodating about their need for modesty. One of the women was weary about such expectations given her negative childbirth experience in Hong Kong, where, according to the participant, every check-up was done by a different doctor, and the patient's preference for female doctors was not always considered. She recalled:

Every country has a different system. Here we have a family doctor to check-up (during pregnancy), but in Hong Kong there is no family doctor. You have to go to the hospital. Every month I had a check-up that (was) usually (done by) a female 
doctor, but once in a while some other (male) doctor would check (me). (Participant 4, Age 38).

She further remembered her postpartum stay at the hospital:

After the birth...that was very horrible, means male doctors used to come and check all postpartum women, you know like vaginal, for the infection or not. I wanted to say, "No, I don't want you to check", but then I had no choice.

The second of the two women, in comparison, had a very positive first experience with her first child, who was born in the United States. She had access to a female OB/GYN throughout and was assisted by an all-female team, including an on-duty midwife for the labour and delivery. Based on her first experience, this participant had similar expectations of health care professionals in Canada.

The fourth participant, newly arrived from Pakistan, expected a female OB/GYN because in Pakistan, which is a predominantly Muslim, and according to the participant, also a very conservative country, where all doctors who specialize in obstetrics and gynecology are female. She assumed that this all-female OB/GYN concept was universal and thus was surprised to discover that, in Canada, she had to request a female doctor, which she eventually managed to secure. Her expectation from that point forth was for a female doctor to see her through to the delivery and post-natal care. She recalls:

So coming here, like I said, I was new, and probably didn't know as much as I know now, or I hadn't had experience, and I maybe didn't ask as much. But my husband is a very conservative person, and he did...I did have a female obstetrician. She looked after me, and even during the process of labour we requested that she be there, and she was. (Participant 3, Age 35).

This recollection sheds light on an additional factor (the role of the husband) in the process. This is the only woman who voluntarily spoke of her husband's involvement, which was not brought up by any other participants. 


\section{Women's expectations of nurses}

The participants' expectations of the nurses were similar across the board. They were mainly looking for the nurses to treat them with care and understanding on a personal and human level. More specifically, the participants expected the nurses to respect the women's need for modesty by ensuring minimal exposure at all times. This was very clearly stated by one of the participants:

My expectation was to have somebody who would be willing to listen to my concerns about modesty, and I am glad to say that my nurses were great. They kept me covered as much as possible, as long as possible. (Participant 3, Age 35).

One of the women also expected the nurses to inform her if the need arose to have a male health care professional or student enter the room or be present for the delivery of her baby. During her first childbirth experience (in Texas) one of the nurses did seek her permission to have a male student observe the delivery, and the participant appreciated this gesture very much, because she had the opportunity to refuse. Her expectation of nurses for her Toronto birth experiences was also the same. However, this did not happen.

Later on, you know, it was really messy and I was hot and I took my scarf off, but the nurses did see my scarf earlier, so they could have asked me that, okay Dr. [so and so] is a man, but...they didn't. (Participant 2, Age 32).

She wanted the nurses to know and understand because of the hijab she was wearing that they should have asked her permission. However, as can be seen from the above statement, the participant was not prepared or informed before a male doctor came in.

When asked about her expectations of Toronto hospitals, one participant stated that she "expected" the nurses to provide her with "good treatment" and ensure that she had "good covering" during vaginal examinations (Participant 1, Age 39). She further believed that the nurses would respect her wishes whenever possible. 


\section{Women's expectations of hospitals}

The general expectation of the participants was that hospitals would be able to cater to their needs regarding modesty during their labour, delivery and post-natal stay by ensuring the women's privacy and by making sure that only female health care professionals would be allowed in their room. Furthermore, if any male presence was necessary, the participants expected to be informed beforehand so they would have time to mentally prepare and cover-up the best they could.

The participant who is also a doctor provided additional insight, from the perspective of her profession, into the general expectations of pregnant women:

The first question that I usually get is... what should I expect in the labour room or the labour ward? I guess their question is, so how many people would be around them, who are these people, if there are any males that would be involved in the whole process, and is there anything, anyway that they can make the whole environment a bit more controlled. (Participant 3, Age 35).

According to this participant, the women try to attain some control in a situation where they are likely to not have any. For them, the best way to make sure that some of their needs regarding modesty are met is by first knowing what they can expect from the hospitals. Then only can they state their expectations and try to compromise, if possible.

A common expectation expressed by all of the women was to have female $\mathrm{OB} / \mathrm{GYNs}$ readily available. This was clearly stated by one of the women when she was asked about her expectations of Toronto hospitals: "I would of course like the hospitals to have more lady gynecologists" (Participant 4, Age 38).

Privacy is another expectation that was spoken of by one of the participants. For her first childbirth experience (in the United States) she had minimal privacy because during her labour she had to share a large room with many other women also in labour. 
Having to share a labour room with multiple women could have been due to economic factors, such as income or the kind of health insurance the participant may have had. Only in the final stages of labour was she taken into a private delivery room. Given this experience, this participant hoped to have private labour rooms in the GTA hospitals. She recalled:

When you got admitted into the labour and delivery, they put you in a big ward...with ten other women, in beds across from each other, and even though they have curtains...I felt that I wasn't having a lot of privacy, because like my husband, the other husbands were there. And the curtains, they keep flying around, you know I didn't feel I had a lot of privacy. (Participant 2, Age 32).

In sum, it is important to note that the underlying expectation of all the women (to be covered and to receive care from exclusively female health care professionals) was the same.

\section{Theme 3: Childbirth and Care Experiences}

This section is about the childbirth experiences of the participants and is divided into the following sub-themes (given that these sub-themes were either spoken of consistently by most participants, or strongly focused on by some): individual childbirth stories chronicling each birth in detail; issues around respect received from health care professionals; and issues around care received from health care professionals.

\section{Individual childbirth stories}

This sub-theme focuses on individual childbirth stories with specific attention paid to the issue of modesty and how this issue was handled by health care professionals from the onset of pregnancy to the post-natal context (i.e. perinatal period). Some of the women have had multiple experiences and each of these experiences warrants individual 
consideration and analysis in order to gain maximum understanding of the complexities that lie therein. Similarly, the stories of the women who gave birth outside of Canada might inform us about equally important childbirth and modesty related issues that exist in other parts of the world, compared to the lessons we can learn from the two participants who have only delivered babies in Canada.

\section{First childbirth experiences}

Participant 1 had her first childbirth experience in a hospital in the GTA. As per her request, she was referred to a hospital that has a high ratio of female patients and to female health care professionals. From the onset of her pregnancy she stated her need for modesty to her female $\mathrm{OB} / \mathrm{GYN}$ based first on her religious beliefs and second on her personal preference. Her doctor assured her that her needs would be met for the duration of her pregnancy and made a special note in her file, thereby validating the special request.

All of the monthly check-ups were conducted by the same doctor and when the labour began the participant was cared for by a team of all-female nurses. The team included an older nurse who ensured that the participant's needs, especially with regards to modesty, were met. The participant recalled:

She was an old lady [and] she knew that I was covering my leg and those stuff (vaginal area) and she told the other nurse, "Careful, you see she's trying to cover, cover her". (Participant 1, Age 39).

This nurse also held the participant's hand and gave her permission to scream, if necessary, just to ease her nervousness. At the time, the participant did not have the ability or time to appreciate this nurse's efforts, but retrospectively has been able to give her the credit and show her the gratitude that she deserved. Her doctor was also instrumental in ensuring the participant's comfort. The doctor allowed the participant to stay in the hospital for a few 
days to be able to participate in the infant-care sessions provided by the hospital, because as a new mom she expressed her worries about not being able to care for her new baby properly. In sum, this participant had only words of praise for her doctor and nurses to express her complete satisfaction with her first childbirth experience.

Participant 2 had her first childbirth experience at a hospital in a big city in Texas. She was a new convert to the religion of Islam due to marriage, and spoke about how through the study of various religious texts overtime, she decided to adopt her faith in practice (behaviour, speech and dress). This participant stated that a female OB/GYN cared for her throughout her pregnancy and an all-female team of nurses and an on-duty midwife assisted her through the delivery process. She said she did not have to ask for female nurses and, in her own words, she felt she was "lucky". The only exception was a male anesthesiologist who administered the epidural. The participant noted that she was fine with this because the procedure only required her back to be exposed, which she felt was "non-invasive" compared to the "invasive" nature of a vaginal examination. The presence of the on-duty midwife was also of significant help to the participant because she was able to deliver the baby in the absence of her OB/GYN.

During the last stage of her labour, a nurse brought in a male student and asked the participant's permission for him to observe. At the time the participant denied permission for the male student, but allowed a female student to take his place. She felt that the nurse asked her permission for two reasons:

The first was because that student wasn't really involved in the birthing process. I am a nursing student now and I know how it works. They had to ask my permission no matter if it was a male or female. And... and I think they saw that I was wearing a scarf, so...they were more careful, to ask me and I felt that they were respecting my religion". 
In retrospect, the participant reported that because she was focused on managing her pain, she would probably not have noticed the presence of a male student in the room. As such, she appreciated that the nurse made the effort to bring the student in front of her so she could process the fact that he was a man and, thus, she was able to say no.

In sum, this participant stated that she had a comfortable and happy overall experience. The only negative aspect in the whole process was the lack of privacy until she was ready to deliver. As such, the participant hoped that every woman should have access to a private room during labour and delivery.

Participant 3 gave birth to her first and only child at a hospital in the GTA. She had been in Canada for only two years before she gave birth. As a medical student in Pakistan, this participant had the opportunity to observe and work on the labour and delivery ward there. She noted a significant difference between observing childbirth from the perspective of a medical student versus experiencing childbirth first-hand.

She noted that in Pakistan, "men are not even allowed near the [labour] rooms...it's all women". As such, she said she did not know that she would have to request a female $\mathrm{OB} / \mathrm{GYN}$ until her husband, who is a "very conservative person", made this request on her behalf. Once the participant understood that things worked very differently in Canada, she took steps to ensure that her modesty was maintained. These steps included informing her doctor and nurses about her need to maintain modesty during labour. Very specifically, she asked "to have [her] thighs covered the whole time, so they were not uncovered". She also asked to be covered "as much as possible, as long as possible, and as soon as possible, after the delivery of placenta". She reported that the nurses were respectful of her wishes and kept her covered accordingly. The participant and her husband also requested the 
obstetrician to be present for the birth. However, during the last stage of the delivery the doctor had to leave to tend to an emergency surgery, so the baby had to ultimately be delivered by a male doctor.

Although disappointed, the couple accepted the change for the following reasons: 1) it was an emergency situation; 2) the baby's well-being was the most important; and 3) the duration of exposure of the vaginal area to the male doctor was very short as it was in the very final stage of delivery. Islamic teachings cite similar reasons for justifying the presence of male doctors during labour and delivery. Overall, the participant reported that she had a satisfactory childbirth experience.

Participant 4 had her first baby in Hong Kong. She said that although she was always strong in her faith, she did not always wear a hijab. She noted that she only committed to wearing a hijab after her first childbirth experience. Furthermore, this participant stated that her need for modesty is primarily based on her socio-cultural upbringing and not so much on her religious beliefs.

It's because of the culture, we have grown up in such a culture, in such a society that we don't like to show our bodies. We were born in such a society that doesn't allow us to show our body parts. Even though it's not because of religion or anything. We have seen our mothers... we have seen our grandmothers...they don't show their body, so it affects us from childhood.

She further confirmed that even if she did not believe in Islam, she would feel the "same" way about maintaining her modesty. Her concept of modesty, therefore, appears to be shaped by various socio-cultural factors.

This participant was the least satisfied with her childbirth experience. She observed that "they [didn't] have that much knowledge about Islam or...modesty in Hong Kong". She further reported that the health care system in Hong Kong did not assign one doctor to 
pregnant women for the duration of their pregnancy. According to the participant, "In Hong Kong, there is no family doctor [for pregnant women...they] have to go to the hospital". Pregnant women were required to have their monthly check-ups done at local hospitals by any doctor that was available. As such, this participant reported that she was examined by multiple doctors, including one male doctor. She said that did not feel that she had a right to request a female doctor. Nevertheless, this participant recalled that even though she was surrounded by male and female health care professionals during the last stage of her labour, the pain was too intense to even care about who was in the room. She stated, "At that time, I was in such pain that I was like, okay...I don't care whether there is male or female...I want to save my life". However, mostly male doctors examined her during a vaginal infection she had following the delivery. She said that she felt helpless because she did not feel that she had the right to refuse or request an alternative. The invasive nature of the examination made her "very, very mad". Ten years later, this anger is still evident in the way she recollected her experience. Furthermore, she could not think of any positive things to say about this experience: "I [don't] have anything positive to say about it". "I would never, never want that thing to happen again".

\section{Second childbirth experiences}

Participant 1 had her second childbirth experience, six years later, at a hospital in the GTA as well. In fact, her satisfaction with her previous experience made her choose to have her second child with the same doctor at the same hospital. This pregnancy was similar to the first until the last month when the participant began to experience early labour. After being admitted to the hospital, she had to undergo an emergency C-section due to the cord twisting around the baby's neck. 
The participant recalled that the doctor informed her of the reason for surgery while on route to the operating room, reassured the participant by saying that she (the doctor) would be present throughout the surgery, and that she would be praying for the participant. The surgery required the participant to be fully sedated and when she regained consciousness, she found her baby beside her.

Once again, she reported that she was highly satisfied with her childbirth experience. She said she was content because she felt that she was in a familiar environment, had zero male presence, had a trustworthy and dependable doctor, and had a caring team of nurses. To this day, the participant keeps in touch with the nurses by visiting them every time she goes to the hospital. The only negative comment she made (when probed) was that the hospital was busier than the first time she was there and so she felt that the nurses could not give her the same attention they did before.

Participant 2 had her second childbirth experience (first, in Canada) in a hospital in the GTA. She had only been in Canada for less than two years when she discovered that she was pregnant. She had been seeing a female gynecologist for general check-ups and felt that she "was very fortunate to find one, because they were not really easy to come by in this city". Her doctor was aware of her need for modesty, but informed the participant that she may not be on-duty the day she goes into labour. Indeed, the doctor was off-duty that day, but the participant was cared for by several, female nurses and midwives, so she felt "comfortable" and "fortunate".

The labour was comparatively short compared to her first, and before long a midwife delivered the baby and the participant felt relieved that she had made it through with zero interaction with male health care professionals. Soon however, she realized that 
a doctor was paged and without prior warning, the male doctor rushed in to deliver the placenta. The participant felt unprepared and did not understand the necessity to have a doctor deliver the placenta. In retrospect, from the perspective of a current nursing student, she realizes the importance of having a doctor examine the placenta to ensure that none of it gets left behind in the womb. Still, she feels that it would have helped her if she were informed about the male doctor beforehand in order to eliminate the surprise factor. She also realized, similar to participant 3 , that this form of male interference was acceptable in Islam because it was an emergency and because no other option was available. In sum, this participant reported that she had a satisfactory experience.

Participant 4 had her second and final childbirth experience in a hospital in Toronto within the first year of her arrival in Canada. She was happy to find a female OB/GYN. However, her happiness was short-lived because she was informed, similar to Participants 2 and 3, that her doctor's presence during labour and delivery depended on whether the doctor was on-duty on the day the participant went into labour. Unfortunately, her labour began at night and only one male doctor was available. She had no choice and thus had to endure another vaginal examination by a male doctor. She recollected, "he was putting the hand inside...it was so disturbing for me".

Ultimately she, similar to Participant 1 , also had to undergo an emergency Csection which, she thought meant less exposure of her vaginal area to the male doctor. For the remainder of her stay in the hospital, she was only checked by female nurses to ensure that her stitches were healing well.

According to the participant, the best part of the whole second childbirth experience for her was the fact that she had, overtime, developed a friendly relationship with the 
female OB/GYN, who provided her prenatal care, so much so that when asked what, if anything, she would not change about any of her childbirth experiences, she promptly referred to this relationship.

\section{Third childbirth experience}

Participant 2 was the only participant who had three childbirth experiences. She discovered she was pregnant with her third child soon after her second was born. Due to the participant's relocation of residence between the two childbirths, her female OB/GYN from her second pregnancy was not accessible. Being fairly new to the country, the participant did not have a driver's license, so she had to rely on her husband to take time off from work in order to drive her to her doctor, which was not possible at that time. She consulted her family doctor to find another female OB/GYN. She recalled, "Actually I remember I said, 'Doctor, please give me a lady doctor'. He said, 'Yes, no problem.”. Two months later, she was finally given an appointment, and to her surprise and disappointment, discovered the doctor was a man. She endured the first check-up primarily for the welfare of the baby because she had not had any check-ups yet.

Later, the family doctor had informed her that he was unable to find a female $\mathrm{OB} / \mathrm{GYN}$ in their area. The participant was upset that the family doctor's office did not mention this when they informed her about the OB/GYN. She was unsuccessful in finding a female $\mathrm{OB} / \mathrm{GYN}$ through her friends and people in her community, and returned to the same male doctor, who seemed to have picked-up on her discomfort, and limited the number of vaginal examinations.

When it came time for the actual birth, the participant had to be induced because the baby was too small. According to the participant, the labour inducing drug resulted in 
rapid and intense contractions that led to a fast delivery. As a result, one of the nurses delivered the baby as the doctor could not reach there in time. The participant remembered, "When the doctor came in, the baby was laying there...so it was nice". She thought that she had avoided the delivery by the male doctor, but as with her second child, the doctor still had to deliver the placenta. This time around however, the participant understood that it was "just for the safety of the mother". She rated her overall experience as semi-satisfactory.

\section{Respect received from health care professionals}

Respect, and in one case, disrespect, emerged as important themes. All of the women spoke of instances in which they received respect from health care professionals during their childbirth experiences. For example, one woman recalled that even in the worst of situations where she had to accept a male OB/GYN for her pre-natal care, the sensitivity and respect that the doctor showed toward her discomfort was sincerely appreciated:

It wasn't very pleasant...but I think ...that doctor knew that...I have the scarf on... so he sort of respected my space as well, and every time, you know, when you go for the regular appointment, after the first time, the female nurse would take your blood pressure and weigh you and the doctor would say, "Okay, everything is normal, just keep doing what you are doing"...he tried to make as little contact as possible. So, it was nice. (Participant 2, Age 32)

The same participant also commended the nurses for all three of her childbirth experiences: "They [the nurses] all seemed to be very culturally competent. And they were respectful" (p. 18).

For another participant, respect meant being treated equally, not differently, by health care professionals. When asked about how the nurses and doctors responded to her need for modesty, she replied, "At that time I used to wear a hijab, but they didn't treat me 
that much in a different way...it was okay, just like normal. (Participant 4, Age 38). To this participant, "normal" meant receiving the same treatment as non-immigrant women, more specifically, non-discriminatory treatment. This woman also found the paramedics who took her to the hospital, as well as the intake nurse who received her initially, to be "nice, polite and respectful".

Another participant spoke of a mutual respect scenario when she referred to how health care professionals handled her request to maintain modesty:

I respected them because they respected my request, that I told them about my problem [with having men care for me]... about my covering thing and those stuff, and they respected that and they helped me about that. (Participant 1, Age 39).

When asked whether she felt her need for modesty was respected, she answered, "It was respected...it was respected, otherwise, I shouldn't have been to that hospital again" (p. 27).

Another participant reported that the one thing she would want to remain unchanged for future childbirth experiences would be the understanding and respectful nurses who, "tried to stick to them (her requirements) as much as possible, and as long as possible" (Participant 3, Age 35).

One of the women encountered two occurrences of disrespect from two nurses (one experienced and one observed). During her post-natal stay at a hospital in the GTA while she recovered from surgery, this participant's baby did not get enough breast-milk. As such, she was given a breast-pump to extract milk and feed the baby from a bottle instead. The following is a recollection of the events that unfolded, in the participant's own words:

She (the nurse) came and she showed me [how to use the breast-pump], but then she was like very rude with me and she said, "Oh, you have to wash that". And I am a Cesarean [patient], I know that for Cesarean women they should take complete rest. And my bed was so high it was a little bit hard for me to get down 
and to go the washroom, and then stand and clean... and she was so rude with me. (Participant 4, Age 38).

She further stated:

And my baby was crying and the crib was higher and a little bit far away, so it was not easy for me to take him from the crib, and I called the nurse and asked her and then she said that, "No, you do it, you have to take" you know, and I was like surprised. It was so painful and I can't even reach the crib and she was saying I have to take my own baby, I have to feed and I have to put back... they won't help me at all. So this is, I don't know, because as an Asian they are treating me like this, or because they have seen hijab...I don't know.

This participant was satisfied with the care she received from the nurses prior to these occurrences. However, encountering this negative experiences, she felt distraught and began to wonder if the nurses behaved similarly with other women as well, or if this behaviour was only directed toward her. The following day the participant witnessed another nurse reacting similarly with another Asian patient who was also recovering from a C-section. She recalled:

They did the same with another woman and that woman also requested the same thing that her baby wants a bottle, and the nurse came and shouted very badly, "No, you have to try, you have to try!" And then the lady, wow she cried so loud that I can hear and then she called her husband and she was talking in Hindi which I can understand and she was complaining the same thing that you see the nurse are treating me like this... and then she was crying and crying. So maybe because she is Asian too, or maybe the nurses behav[iour] is like that (p.30).

Having witnessed two such incidences from two nurses, toward two Asian women, this participant wondered the nurses' behaviour was a form of discrimination. However, she was cautious about labeling it as such since she also felt it could be part of the nurse's nature.

\section{Care received from health care professionals}

Although care can be seen as a form of respect, and vice versa, I have presented these as two separate sub-themes. My rationale for this is based on the fact that the 
participants themselves appeared to differentiate between the two. Three participants chose to use the word 'respect' consistently throughout the interview, whereas one participant chose to highlight the word 'care'. So, although this theme was only spoken of by one participant, it still deserves acknowledgement because it meant a lot to her. This woman focused on this aspect of care in great detail and chronicled her experiences with her doctor and two nurses in particular, who, the participant felt, went that extra mile and provided the kind of care that exceeded their duty. The following quotes reflect some of these experiences in the participant's own words.

Care experience 1: First labour at a hospital in the GTA

During the labour and delivery, the participant recalled that one nurse, helped her through the process.

When, like my nurse, who was with me that time, I won't forget that she held me when she saw my face that I was nervous about giving birth right? She says..."I know you feel bad, you feel scared, you can scream at me, I won't mind. If you wanna hold my hand, scratch me, I won't mind", I won't forget. (Participant 1, Age 39).

This participant further stated:

She was holding my one hand and she was talking to me like the way a mom should talk to the child. She was an old lady as I mentioned. The way she was talking, she knows that I was covering like my. The way she was telling (other nurses to cover me) looked like she's taking care of her own child. And my doctor was laughing there, she knows. I won't forget that. There are people, still now we know in the world that they do care for the patients, for others. When they do care, they show them the love that people will feel that they are their own people. They won't feel that they are patients there, right?

Fifteen years later, the participant still visits that nurse from time to time and during their last encounter the nurse hugged her son and said, "I'm the first one who held you. I knew you before you were born. I'm as old as your grandma". The participant fondly remembered every interaction she had with this nurse. 
During her post-natal stay at the hospital, another nurse went out of her way to help this participant reach and feed her newborn and also kept her company in the evenings.

There is a nursery room there obviously, when patient can't sleep, [the nurses] come and take the baby (to the nursery), right? But obviously when I'm a first [time] mom, I want my baby with me. And at the same time, if baby is crying, I can't take care of him. So, eventually a nurse came, and I was surprised that she held my baby and sat right beside me so I can see the baby. I was there five night[s], those five night[s], most of the time she was with me. Every few minutes when she saw that I'm awake, she says "Wanna see his face, wanna hold him?" She gave me the baby. After a few minutes, she took the baby, because she knew by watching my face that it was hurting me. So... I don't think that comes from the duty, it comes from the feelings. They feel it, and that's what I respect them for..

The participant felt that the care she received exceeded the nurses' job requirements and further, that the ability to provide such care is part of one's intrinsic nature.

\section{Theme 4: Recommendations}

This theme is significant to me because it was based on the ideas voluntarily brought up by the participants. The recommendations were based on their post-childbirth reflections, and can be divided into recommendations for: 1) the health care system;2) health care professionals (doctors and nurses); 3) students; and 4) other pregnant women.

\section{Recommendations for the health care system}

Three women voluntarily offered their recommendations for bettering the health care system's ability to cater to the needs of any women (not limited to Muslims) who value modesty. Modesty emerged as a theme that is considered important by women, in general, on one or more of the following levels: personal, cultural and/or religious. One of the participants viewed modesty in childbirth from a religious lens when she stated, "I know that so far, even in Christianity, there are a lot of women... who prefer modesty. 
They even give childbirth at home, you know, through a midwife" (Participant 4, Age 38). Given this information, this participant felt that one way in which the health care system can accommodate the needs of women who have issues around modesty is by working toward recruiting more female doctors who specialize in obstetrics and gynecology. She believed that every child-birthing woman should have the right to choose a female doctor regardless of when she delivers.

Another participant who also shared this belief further recommended that the health care system should provide more funding for midwives, so they too can be readily available for women who need them. She was so content with her experience with midwives that she stated that she preferred midwives over doctors and provided the following reason:

"[midwives are] more sensitive, more softer... and doctors are doctors, they are clinical, they are cold". (Participant 2, Age 32).

Based on her personal experience of being unable to be referred to a female $\mathrm{OB} / \mathrm{GYN}$ because none could be found in her area, this participant noted that there is a definite need for more female OB/GYNs so more female medical students should be encouraged to pursue this specialization. She also suggested that more female medical students should be encouraged to become General Practitioners as well, given that some women do not want to be examined by male health care professionals for non-childbirth related care as well. The following is an example of what she meant:

Suppose an elderly lady came in with a broken leg and she didn't feel comfortable lifting up, you know her pants for the male doctor to look at her legs, so...I feel that we need more female doctors, not just for $O B$ purpose, but for...you know, general purpose. (Participant 2, Age 32).

Although the participant who is a doctor was the only one who did not voluntarily propose any similar recommendations, she still corroborated what Participant 2 said by 
mentioning that in her interactions with pregnant patients, modesty is frequently brought up and is not limited to Muslim women. She stated, "I've seen many women, many nonMuslim women, who have the same request and same issues" (Participant 3, Age 35). The same participant noted that if hospitals can accommodate women who wish to give birth in a room full of relatives with video cameras and so on, then the needs of women to maintain their modesty should also be easy to accommodate.

A long term recommendation offered by another participant included encouraging the health care system to build more hospitals (since her second birth experience, albeit wonderful was also very crowded) and hire more staff, especially female staff to cater to the needs of any woman who presents modesty as an issue. She specified that the hospitals should be good hospitals, like the one she gave birth in, and unlike the other hospitals that she encountered for non-childbirth purposes where she felt like a "burden" and voiceless. (Participant 1, Age 39).

\section{Recommendations for health care professionals (doctors and nurses)}

Some of the participants offered specific recommendations for health care professionals aside from those suggested for the system at large. For example, one of the participants (the nursing student) observed that:

[Although] they are teaching doctors and nurses about cultural sensitivity and everything, but um... a lot of health care professionals still don't know. They still don't really care about Muslim women and everything. Like, for instance, when I am on the unit and I see that sometimes, you know like the patient is a Muslim, and then the nurse manager, she will assign a male nurse to take care of that woman. Or the clinical teacher will assign a male student to take care of that woman, and you know that they're gonna say no. They know that she has a scarf on...the whole family has a scarf on. Just why don't you think before you assign the nurse to the patient? They really should take care of that. A lot of times they overlook that. (Participant 2, Age 32). 
This participant recommended health care professionals to be more aware and considerate of the needs of their patients by putting theory (what they are taught) into practice (what they do).

From her experience as a woman who has given birth, the same participant recommended that doctors and nurses should be more like the ones whom she encountered during her pregnancies. She referred to the nurse who asked her permission before allowing a student to observe, and the male OB/GYN who showed sensitivity to her discomfort by limiting the number of examinations.

\section{Recommendations for students}

The single underlying thought within this sub-theme was that more female medical students should choose to specialize in obstetrics and gynecology. Three of the participants agreed, including the doctor when she spoke from her personal experience, that although

- they understood why their doctors could not guarantee their presence, they should have been able to guarantee them the presence of other female doctors. One participant, in particular, was a significant advocate for this recommendation and stated, "Why, if [male doctors] can be on-duty then why the women doctors can't be on-duty?" Participant 4, Age 38). No recommendations were made for nursing students.

\section{Recommendations for other pregnant women}

This sub-theme presents recommendations that were made by the participants for any pregnant women who may be concerned about modesty, including themselves, for any experiences with childbirth which they may have in the future. One participant blended her experiences of being a doctor and a woman who has experienced childbirth to present the following recommendations: 
1. Present a birth plan to their obstetrician that prioritizes their baby's wellbeing and their need to maintain modesty over all else.

2. Communicate their need to maintain modesty as clearly and specifically as possible to their obstetrician (at the onset of the pregnancy) and to the nurses (when they are admitted into the hospital). Specifics should include their requirement to ensure their female obstetrician's presence as well as their need to be covered as much as possible, for as long as possible, and as quickly as possible after delivery.

3. They could also try to see if they can schedule their delivery (be induced), because that would increase their chances of having their own obstetrician present.

4. They can seek out midwives and birth attendants. With this option they have to be aware that in case of complications during childbirth, a doctor will have to intervene and that the doctor can be either male or female.

5. (This applies to Muslim women only) If all their efforts fail and the baby has to be delivered by a male doctor, they (the women) should remember that Islam has allowances for this scenario granted that the situation is an emergency and/or there is no other option available. In Islam, saving life is considered more important that maintaining modesty.

The planning that is suggested by this participant is reflected in the interviews of Participants 2 and 4 as well, both of whom recommended better preparation on the part of the pregnant women. For participant two, this preparation included: 1) insisting upon a female $\mathrm{OB} / \mathrm{GYN} ; 2$ ) if option one fails, "getting a female midwife to see [her] through the whole pregnancy"; 3) if option two fails, doing her own research to find a female OB/GYN any where in the GTA because she is now able (has access to transportation), and would 
not mind traveling a longer distance to fulfill this requirement ; 4) as a last resort, requesting the doctor to have her labour induced on a day when the doctor would be oncall; and 5)"keeping [her] scarf on throughout [the labour and delivery process]" with the hope that health care professionals caring for her would understand her need for modesty.

For participant 4 , the planning to ensure modesty during delivery would include the first two options as stated by Participant 2 . However, if she is unsuccessful in securing either a female OB/GYN or a female midwife, she would,

...rather go back home (to Bangladesh) and do the delivery, because in Bangladesh there are always gynecologists, all are women, so a woman will deliver your baby for sure. (Participant 4, Age 38).

This participant further stated that her need to ensure a team of exclusively female health care professionals would be important enough to sacrifice the benefits of the Canadian health care system. According to this participant, she would only decide to remain in Canada if she were informed beforehand that either her baby's or her own life may be at risk.

Participant 3 , in contrast to Participant 4 , stated that although her home country (Pakistan) also has all female staff in the labour and delivery ward at all hospitals, she would prefer to deliver in Canada because, "everything here (in Canada) is patientcentered, whereas there (in Pakistan), the resources are limited" and even though the nurses are all women, "their numbers are limited also". As such, the care she and her baby would receive would be compromised. This participant would, thus, prioritize her need to receive care over her need to guarantee modesty. 


\section{DISCUSSION}

The qualitative analysis of immigrant Muslim women's views regarding modesty in the specific context of childbirth in Canada revealed a number of key findings.

All of the participants view modesty from both a religious as well a personal lens. Modesty was seen as a significant part of their day to day lives and was perceived to be reflected in every aspect of their lives including behaviour, speech and dress. This finding is in accordance with Islamic texts such as the Hadith of Sahih-al Bukhari, which states that modesty is reflected in a Muslim's speech, dress and conduct. Arif (2006) further affirms that in Islam, "the Qur' an urge[s] all Muslims to show respect and maintain modesty in thought and deed" (p.32).

Although all of the participants in this study spoke about adhering to the basic tenets of Islamic etiquette in behaviour and in speech, the women's interpretations differed regarding the dress. For two of the participants, covering their hair was equally important as covering the bodies, but for the two other women, covering the body was more important than covering the hair. This illustrates that women giving birth can also have different ideas of covering, thus need to be assessed individually by health care professionals. This view is supported by the two theoretical frameworks that guided the study, which emphasizes the need for health care professionals to not only develop and understanding and awareness of their client's culture and provide care accordingly (ie., cultural competence), but also to delve deeper and find out how the individual client perceives her/his culture (ie., the importance of paying attention to the intersctionalities of identities). For example, it is possible that a woman who wears a hijab may be less concerned about being examined by a male doctor than a woman who does not wear a 
hijab. An article by Hutchinson and Baqi-Aziz (1994) that offers suggestions for nurses on how to care for pregnant Muslim women corroborates this finding of the differing individual requirements of patients. The authors state, "Modesty needs and preferences vary and require individualized assessments and planning to minimize family stress and staff-family conflicts" (p. 769). The participant in my study who did not mind having a male anesthesiologist administer the epidural because she felt that exposing the back was not as invasive as exposing the vaginal area, is one example of how individual ideas of modesty, even within such a small sample, can differ. While the rest of the participants insisted on zero male presence, this participant was the only one who stated that she would allow some male presence as long as the contact was restricted to procedures that did not require vaginal exposure.

Sperstad and Werner (2005) also believe that although it may be useful for health care professionals to rely on general information about various cultures and practices, they should also be careful not to stereotype, because the perceptions of individual patients from similar cultures may differ. This is similar to the findings of Fenwick, Hauck, Downie and Butt's (2005) study that the individual needs and expectations of pregnant Australian women differed significantly from one another.

Modesty as a product of socio-cultural upbringing emerged as another theme. Initially, the impetus for this study was primarily driven by the religious underpinning of modesty. However, the findings revealed that for most of the participants, their concept of modesty was seen as the product of their culture and society. For these participants, religious perceptions regarding modesty were secondary. This is a new finding that has not been suggested in any other literature that I have reviewed. However, it is important to 
consider the following: is it possible that the participants' "cultural values" stem from the long-standing religious beliefs and practices passed-on through the generations? Consider, for example, the participant who stated that even if she was not a Muslim, she would still have the same issues regarding modesty as she does now, because that is what was rolemodeled for her by her mother and grandmother. So how is culture influenced or shaped by the dominant religion of a country? This question requires exploration in a future study.

The findings further revealed that some of the participants reported growing up in a culture and society in which people, especially women, guarded their modesty, so much so, that, as two of the participants informed, the labour and delivery wards in their home countries do not allow men to be on the premises. For example, childbirth, in Bangladesh and Pakistan is considered a female-only event where even the thought of being examined by a man, as one woman informed, is "unthinkable" and "beyond imagination". This notion is corroborated by Callister and Vega (1998) whose study of pregnant Guatemalan women, in Guatemala, found that they (the participants) sought limited professional care during their pregnancies, because "they may not have felt comfortable with [vaginal] examinations", which would require the participants to compromise their modesty and privacy, especially in the presence of men (p. 291).

The findings of the current study also revealed that the participants wanted hospitals, doctors and nurses to meet their collective (culture competence) as well as individual expectations (intesectionalities of identities) regarding their need to maintain modesty. The participants expectations in this regard included two key aspects: a) to ensure that they are cared for by exclusively female health care professionals, without the interference of any male health care professionals during the prenatal (pregnancy), 
perinatal (labour and delivery) and post-natal (after delivery) periods, and b) to remain covered as much as possible, for as long as possible and as soon as possible, after delivery, to ensure minimal exposure of their bodies. Both of these factors are further explored below.

The need to be cared for by an exclusively female team of health care professionals begins with finding a female OB/GYN. This preference for a female OB/GYN within the Muslim population was also found in other studies, including one by Reitmanova and Gustafson (2008) whose study, based on the maternal health and care needs of immigrant Muslim women in St. John's, Newfoundland, determined that all of the participants had a strong preference for having a female attendant care for them during their childbirth experience, and agreed that having a male physician would only be acceptable if there were no other options. The participants of this current study also pose the same clause under which they would allow a male doctor to deliver their babies. An additional clause also raised by the participants of the current study under which they would accept a male doctor would be in the case of an emergency.

A study by Maimbolwa, Yamba, Diwan and Ransjo Arvidson (2003) conducted in Zambia also found that one of the main reasons for delivering children at home with the assistance of midwives was to eliminate the possibility of having their modesty breached in the presence of men. Another study conducted in Lebanon by Kabakian-Kasholian, Campbell, Shediac-Rizkallah and Ghorayeb (2000) explored Lebanese women's reactions to the medical management of their pregnancies. The findings of the study revealed,

All of the participants preferred female obstetricians or midwives to their male counterparts. The main reasons given for this preference were modesty toward male physicians and ease in communicating very personal issues with a female physician" (p.106). 
The latter reason is an additional piece of information that was not provided by the participants in the current study.

It is important to address that the preference for female health care professionals is not limited to the Muslim population in Canada, because it is also prevalent on a universal scale with Muslims and non-Muslims alike (albeit for different reasons). This notion is supported by several studies, including one conducted in the United States by Lurie, Margolis, McGovern, Mink and Slater (1997) that revealed that a significant number of women (from the general U.S. population) preferred and felt more comfortable about being screened for breast and cervical cancer by female physicians. Two Dutch studies also provided further support this view. The first study by Bensing, Den Brink-Muinen, and De Bakker (1993) found that, for various reasons, female patients (from the general population) tended to choose female general practitioners, especially for female-specific problems. The second study by Kerssens, Bensing, and Andela (1997), also had similar findings to the first Dutch study, and further informed,

The majority of [women] who prefer female health care professionals indicate that they talk more easily to females that to males, and feel more at ease during internal examinations by females than by males (Abstract, p.1531).

Furthermore, this preference for female doctors and nurses often goes hand-in-hand with women's need to cover during the childbirth experience, a factor that also exists in some non-Muslim populations. Roberts (2002) described childbirth as an experience that represents a special time for individuals of all religions. Arif (2006) corroborates that this spiritual belief in modesty exists in all major world religions that expect "their followers to adopt some aspect of modesty, such as covering the body or head and limiting social interaction with the opposite sex" (p.32). Some of the participants in the current study also 
spoke of this multi-spiritual aspect of modesty. The participant who is also a doctor stated that modesty was one of the primary concerns presented to her by pregnant Muslim, as well as non-Muslim women. Another participant corroborated that she also knew of many Christian women who guard their modesty during childbirth. De Sevo (1997) also noted that one of the Jewish traditions during childbirth includes the need to have the childbirthing woman's head covered. The need to cover, thus, appears to have religious underpinnings in several major religions.

The need to cover and remain covered for the duration of labour and delivery was another key finding in this current study. Each participant addressed this issue of covering as necessary part of their childbirth experience, which they expected health care professionals to understand and respect. This is reminiscent of the cultural competence approach of nursing care that requires health care professionals to develop in-depth understanding of the cultures, and the differences that lie within cultures. An American article by Lawrence and Rozmus (2001) deals specifically with the issue of providing culturally sensitive care for the general Muslim patient and suggests, "Nurses should be careful to maintain the patient's modesty during examinations and procedures, screen the patient from view, and uncover only the part of the body being examined" (p. 230).

Hutchinson and Baqi-Aziz's (1994) article focuses specifically on women giving birth to further address this need for screening the patient from view by stating, "Whenever possible, a private room is preferable. [However if this is not possible], in a semi-private room, the Muslim woman should be given the bed farthest from the door, with privacy screens or curtains" (p. 770). For one of the participants in the current study, covering meant privacy as well. However, this participant noted that curtains that separated her 
while she was in labour, from the ten other women who were also in labour, did not suffice because [the curtains] "kept flying around". In accordance with the intersectionalities of influences framework, this finding is a good example of the fact "that immigrant women [do indeed] have unique ideas as to what is important to them and what needs to be done to transform their lives in health-promoting ways" (Guruge \& Khanlou, 2004, p.43).

The participants in my study also expressed their wish to have more control over the details of their childbirth experience. They stated that they wanted their concerns to be heard by their OB/GYNs, they wanted to be able to control, as much as they could, the number and gender of health care professionals that are present in the delivery room. This finding is corroborated by several authors. As noted in the Literature review chapter, Leap (2009) is an advocate of replacing 'women-centered' care with the practice of 'womancentered' care, with the objective to ensure that individual women possess primary control of their childbirth experiences. This practice is necessary because Simkin (1991) found that the amount of control that women had over their childbirth experiences had a positive correlation with the satisfaction they felt during those experiences. This satisfaction, in turn, provided them with positive memories of the births of their children, a finding that is substantiated by the current study.

When asked about their ideal childbirth experience, three of the participants listed several ideas. Some of these ideas included preparing and presenting a birth plan, researching resources to increase their chances of finding a female $\mathrm{OB} / \mathrm{GYN}$, and moving to their home-country to ensure that their need to have a female doctor deliver the baby was guaranteed. All of these ideas illustrate the participants' need to have more control over their individual childbirth experiences. 
Giving birth in a foreign country was a common factor for all of the participants in the current study. For three of the women, they experienced childbirth within the first three years of immigrating to Canada. Collins (2008) notes that many newcomers to Canada who come from "countries where spirituality is assumed to be an integral part of health and healing, [find that the] current health care system does not often recognize the role of the spiritual" (p.89). Although Collins refers to spirituality as being more than a part of an organized religion, for the purpose of this study, I shall focus only on the aspect of religion. In the current study, only one participant verbally indicated, to her doctor from the onset of her first pregnancy in Canada, that her need for modesty was partially based on her religion. The other three participants presented modesty only as a personal choice and did not verbally involve their religion. However, of the latter three participants, two wore hijabs, which symbolically drew attention to their religion. For the most part, all of the participants felt that their need for modesty was respected, regardless of whether the health care professionals understood the women's preferences to be based on their religious or personal beliefs. This finding is supported by Collins and Guruge (2008) who state,

Some health care professionals have embraced a culturally sensitive approach to their practice that is based on a multicultural perspective. With this approach, health care professionals provide care that acknowledges and respects the cultures and cultural practices of their clients, and tend to develop awareness of their own cultural values and beliefs that shape health care encounters (pp. 21-22).

As further noted by Guruge and Collins (2008), simply embracing a culturally sensitive approach, however, is not sufficient. Caring for diverse clients requires health care professionals to be culturally competent. The word 'sensitive' indicates the need to react to or be aware of the patient's requests, whereas 'competent' refers to the ability of health care professionals to be knowledgeable about and respond to the diverse needs of their 
clients, first based on general cultural information and second, by combining the general information with specific information about the client based on the individual cues that the latter may provide. Adopting the cultural competence approach along with an intersectionalities framework is thus necessary to foster positive client-practitioner relationships.

The highlights of the experiences of the participants in the current study included doctors and nurses who were not only caring and respectful of the women's cultural and religious beliefs throughout the course of their pregnancies, but were also actively involved in determining the specific needs of each of the patients under their care. When asked about the best part of their individual childbirth experiences or an aspect of their experiences that they would not change for future childbirths, they all spoke of either their relationship with their doctors and/or their interaction with the nurses and midwives. The only exceptions were posed by two participants. The first participant referred to an experience she had in a non-childbirth context at a hospital in Canada that was different form the one she gave birth in, where she reported that one of the health care professionals treated her like a "burden". The second participant referred to her post-natal stay at a hospital in the GTA where she observed two nurses speaking rudely and refusing to help her, as well as another patient when they needed assistance. This participant felt that the nurses' behaviour may have been directed specifically to people who are from the participant's culture.

Of the eight childbirth experiences that were cumulatively captured in this study, six occurred in Canada. Of the six, one participant rated both her childbirth experiences as highly satisfactory, whereas the other three participants rated their childbirth experiences in 
Canada as satisfactory. The primary reason for this difference in rating (highly satisfactory vs. satisfactory), according to the participants, was the fact that ultimately all of their experiences had to involve a male physician. However, they all understood that this involvement, regardless of their personal discomfort, was necessary and therefore allowable in Islam. This finding is supported by The Fatwa Center of the Islamweb website formulates fatwas (laws or rules) about various aspects of everyday life based on the teachings of the Qur'an and Hadith. Fatwa 82093 (1999) specifically addresses the issue of Muslim women being examined by male doctors and states that Muslim women (by Islamic law) cannot expose any part of her body (with the exception of her face and hands) to any man. And further, that the only reason under which a Muslim woman would be allowed to remove her cover in front of a male doctor is if the situation is such that delaying treatment may put the woman's life (or the baby's life, in the case of the current study) at risk. This allowance, according to Islam, provided the participants in this study with some consolation, however, it was not able to ease their personal discomfort.

One of the problems faced by some of the participants was the inability to find or access a female $\mathrm{OB} / \mathrm{GYN}$. One participant had a particularly difficult time with this issue. Hutchinson and Baqi-Aziz (1994) focused on this issue in their article that offers nursing care advice for the "childbearing Muslim family" to state:

During the prenatal period, finding and obtaining quality care is one of the most important needs of all childbearing families. Muslim women who are unfamiliar with the health care resources in their area may have to rely on word-of-mouth referrals, which could delay their obtaining prenatal care. If they are unable to drive, or are unfamiliar with the area, these women may have to rely on their husbands to get them to their appointments (p.768).

The situation described above is similar to what happened to one of the women in this study. Due to the participant's relocation of residence, her previous female OB/GYN's 
office was too far for her to access. This participant, who did not have a driver's license was limited number of physicians who had offices in close proximity to her home. This participant, (similar to the situation described by Hutchinson and Baqi-Aziz), sought wordof-mouth referrals, which led her to only one female OB/GYN who did not accept any new patients. The overall situation led this participant to question whether there were enough female OB/GYNs in Ontario.

As noted in the Literature review chapter of this study, The College of Physicians and Surgeons of Ontario (CPSO) reported that, as of August 7, 2010, there were a total of 206 doctors in Ontario who specialize in obstetrics and gynecology, 100 of which are women. As such, it should not be difficult for pregnant women to find a female OB/GYN. It is important to mention here, however, that it has been six years since this participant had this experience, so the current numbers of OB/GYNs might not provide an accurate picture of the situation in 2004. The participants speculated that even if the numbers of existing male and female OB/GYNs is almost equal, the issue could be that the demand for female $\mathrm{OB} / \mathrm{GYNs}$ was higher than that of their male counterparts. This is a realistic possibility. However, it is also possible that major cities, such as Toronto, have a higher concentration of female OB/GYNs than smaller cities.

The findings also revealed that the participants exhibited a preference for midwives. This preference was influenced by two factors: a) midwives are more readily available than OB/GYNs, and b) midwives are mostly women. According to the website of The Association of Ontario Midwives (2010), there are approximately 500 practicing midwives in Ontario, which is five times more than the number of female OB/GYNs available in Ontario. 
According to the preganancy-info.net website (2009), "[a midwife] is almost always a woman" (II 1).

One of the participants, the only one who had an on-duty midwife in the labour room for one of her childbirth experiences, further articulated that midwives are softer and gentler than doctors. She attributed this difference to the fact that doctors have various concerns to deal with, whereas midwives are only concerned with the woman under their care. In an ethnographic study of child-birthing women's experiences with midwives in England, Walsh (1999) found that perceptions of women giving birth were significantly influenced by their relationship with their midwives, and that the participants referred to their midwives as "friends". This positive client-practitioner relationship is the essence of the cultural competence framework and these findings are a testament to the benefits of this approach.

Although Walsh's (1999) study referred specifically to midwives, the participants of the current study reported that they valued the relationship they had built with their doctors, and similar to the participants in Walsh's study, the women in the current study also referred to their doctors as "friends", and viewed these friendships as positive aspects of their childbirth experiences. The participants further stated that this "friendly" relationship made them feel more comfortable to share their personal thoughts and concerns with their doctors. This finding shows that, contrary to the views previously expressed by one of the participants, this ability to develop friendships with the women under their care is not limited to midwives, and exists in doctors as well. This comfort in communicating with their primary care providers is an important issue with all pregnant women, and especially so for new immigrants who might still be in the process of building new friendships. 
In summary, I found both the cultural competence and the intersectionality of identities frameworks have helped me to understand the findings that my study has generated. I have arrived at the conclusion that the overall objective for health care professionals should be to find a balance between the objectives proposed by both frameworks in order to gain awareness of and show respect for the clients' culture in a collective sense, but also to seek out and cater to the individual differences that exist within the collective.

\section{Study strengths}

This study sought to understand the individual views and experiences of modesty during childbirth. Certain life events, such as childbirth, are experienced individually, not collectively, and thus are subject to the individual expectations and needs which can be influenced by a variety of factors. These factors include, but are not limited to, the way each woman has experienced and gained understanding of their culture, religion and ethnicity and society. I believe that the pre-existing personal relationship between the participants and I enabled the participants to share information more comfortably, freely and openly than if they were to be interviewed by someone they did not know. This nature of collection of information is a strength of this study. Also, as far as I know, this is the first Canadian study that deals specifically with the need for Muslim women to maintain their modesty during childbirth.

\section{Study Limitations}

Using interviews as a data collection tool has the following drawbacks, as noted by Creswell (2004): 1) gives filtered information that are subjective in nature and therefore open to biases and minimizes trustworthiness; 2) the researcher's presence may further bias 
the participants' responses; and 3) participants may have difficulty articulating responses. To combat the first drawback, I rephrased questions in several ways to elicit a less-filtered response. Since the repetitive nature of this process was successful in drawing out similar responses, the level of trustworthiness was increased. For the second drawback, I used my existing rapport to establish a level of comfort during the course of the interview. To tackle the third limitation, I asked simple questions and used words that the participants used to ask for clarification and expansion. Also, the semi-structured nature of the interviews enabled me to use open-ended questions in order to obtain clearer responses.

I used a convenient sample and its size was very small. Further work with a larger sample is recommended to gain a more in-depth understanding of the topic and to reflect the diverse needs and perceptions of individual child-birthing women. 


\section{RECOMMENDATIONS}

This section is comprised of recommendations provided by the participants of this study, as well as myself, on how to address the issues brought forth by the individual and collective childbirth experiences of the participants. These are focused on the individual women, health care professionals and the health care system.

The participants' recommendations for other women and for themselves for any future childbirth experiences they may have included seeking out resources, and presenting birth plans to their $\mathrm{OB} / \mathrm{GYNs}$ to help them understand the individual needs of their patients better.

Recommendations for the health care system were focused on the need for the recruitment of more female $\mathrm{OB} / \mathrm{GYNs}$ and general practitioners as well, with the intention of meeting the modesty needs of any woman who requires it, based on personal preference.

- Recommendations for the health care system also included the need for increased funding for midwives, who perhaps can be more readily available for patients.

Recommendations for health care professionals included the need for doctors and nurses to be better aware of the needs of the diverse women who give birth under their care. Although most of the participants' childbirth experiences were positive, the women felt that their experiences could have been better if some of the doctors and nurses were more diligent in respecting the needs of the participants. For example, health care professionals can seek permission before entering the labour room, so the participants would have a chance to cover themselves. Recommendations were also made for teaching hospital staff to be more cognizant of the nursing and medical students they assign to certain patients, and to ask permission of individual patients before assigning students to 
care for them. Recommendations were also provided to encourage and provide more incentives for more female medical students to choose to specialize in obstetrics and gynecology.

I join the participants in advocating to implement all of the recommendations listed above.

\section{Implications}

The implications of this study are two-fold. On one hand, this study shows that the majority of the childbirth experiences explored were positive. In this regard, the findings of this study can be seen as applause for the majority of health care professionals who are providing more than satisfactory care for the patients under their care. On the other hand, this study also shows the gaps in the current health care system. These gaps range from the unavailability of female OB/GYNs to provide care for women throughout their pregnancies, to a few health care professionals who sometimes ignore the needs of individual women under their care. Most of the participants in this study were examined by male doctors, on some level, which the participants felt compromised their dignity and emotional wellbeing. As such, this study's findings help inform health care professionals and the current health care of the gap that still between the talk about delivering culturally relevant, individualized care and the actual practice of delivering such care. 


\section{CONCLUSION}

This study addressed the overarching question: What are Muslim women's views toward, and experiences of, maintaining modesty during childbirth?

This study's findings show that while the views of participants towards modesty sometimes differed, their views toward modesty in the specific context of childbirth were similar across the board, in that they all wanted to be cared for by exclusively female health care professionals and they all wanted to have their bodies covered as much as possible and for as long as possible during their childbirth experiences. Given the negative experience I had at a GTA hospital during the delivery of my child, I assumed that other women might have faced similar negative experiences. However, this study has shown that, in most instances, the participants' values and needs regarding maintaining their modesty throughout their childbirth experiences were satisfactorily met. Gaps do exist, however, requiring improvements to the health care system, such as, not having sufficient female $\mathrm{OB} / \mathrm{GYNs}$ to meet the needs of the growing population of immigrant women.

Further exploration into the topic with a larger sample of Muslim immigrant women in Canada is recommended to learn more in-depth about this topic. 
APPENDICES 


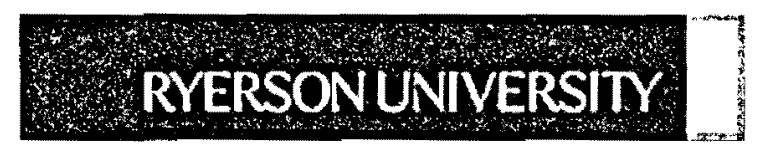

\section{APPENDIX A: \\ Information and Consent Agreement for Participants}

Dear potential participants,

My name is Sanjana Khan. I am a graduate student in the Master of Early Childhood Studies program at Ryerson University. As part of my requirement to complete this program of study, I am attempting to work on a Major Research Project with the intention to explore Muslim women's views toward modesty and maintaining modesty during childbirth. I am inviting you to participate in this process, with the intention to increase awareness and understanding of the needs of Muslim women during the childbirth experience.

It is important that you read the following information and ask any questions necessary to be sure you understand what you will be asked to do. You will also have the opportunity to ask questions about the project before you make your decision about participating. Your participation in this study is voluntary; you are under no obligation to participate. You have the right to withdraw at any time. If you decide not to participate, rest assured it will not have any impact on your existing relationship with the investigator, or any future relationship you may have with Ryerson University.

\section{Investigator:}

Sanjana Khan

Graduate student, School of Early Childhood Education

Ryerson University

350 Victoria Street

Toronto, Ontario

Canada M5B 2K3

Telephone Number: 416-300-2646

E-mail:s27khan@ryerson.ca

\section{Research Supervisor:}

Sepali Guruge, $\mathrm{RN}, \mathrm{PhD}$

Associate Professor, Faculty of Nursing

Ryerson University

350 Victoria Street

Toronto, Ontario

Canada M5B 2K3

Phone: 416-979-5000 ext.4964

E-mail: sguruge@ryerson.ca

Study Title: Modesty during Childbirth: Muslim Women's Perspectives. 
Study Location: Your home.

\section{Description of the Process:}

The procedure will require me to engage in one face-to-face interview. The interview will be a maximum of two hours in length and will be tape-recorded. The tapes will only be accessible by me and my supervisor. The tapes will be transcribed and pseudonyms will be used to ensure your privacy and confidentiality. At the end of the study, the tapes will be destroyed.

\section{What is Experimental in this Study?}

None of the procedures are experimental in nature.

\section{Risks or Discomforts:}

This study will require you to recall your birth experiences, which may at times be emotionally stressful for you. If this happens, you have the right to refuse to answer a specific question or choose to respond to it later.

\section{Benefits of the Project:}

Participating in this project will allow you to share your view of modesty and maintaining modesty during childbirth, which will inform health care professionals so they can deliver more culturally and spiritually sensitive care in all hospitals across the Greater Toronto Area.

On a personal level, you will have the opportunity to share your stories in a safe, comfortable and confidential environment. This sharing may lead you to experience emotional fulfillment and a sense of personal accomplishment for contributing to a study that may have positive implications for women in situations similar to yours.

\section{Questions about the Project/Study:}

If you have any questions about the project now, please ask. If you have questions later about the project you may contact me by telephone or e-mail at:

Telephone Number: 416-754-1952

E-mail: s27khan@ryerson.ca

If you have questions regarding your rights as a human subject and participant in this project, you may contact the Ryerson University Research Ethics Board for information.

Research Ethics Board

c/o Office of the Vice President, Research and Innovation

Ryerson University

350 Victoria Street

Toronto, ON M5B 2K3

416-979-5042 


\section{Agreement:}

If you wish to participate in this study, please confirm by signing below. Your signature indicates that you have read the information in this agreement and have had a chance to ask any questions you have about the project.

You have been given a copy of this agreement to keep.

You have been told that by signing this consent agreement you are not giving up any of your legal rights.

Signature of Participant

Date

Signature of Investigator

Date

My signature below indicates that I voluntarily agree to the audio-taping of my interview.

Name of Participant

Signature of Participant

I confirm that I have explained the nature and purpose of the study to the person named above. I have answered all questions.

Signature of Investigator

Date 


\section{APPENDIX B:}

Interview Guide Questions for Participants

Date of interview:

Time:

Place:

Interviewer:

Interviewee:

1. What is your idea of modesty?

2. Where were your children born?

3. Did you give birth at home or at a hospital?

4. When you think about your childbirth experience, how do you feel?

5. In what ways did your childbirth experience make you feel satisfied?

6. In what ways did your childbirth experience make you feel not so satisfied?

7. In what ways would you like your childbirth experience to have been different?

8. In what ways would you like your childbirth experience to have been the same? 


\section{APPENDIX C:}

My working notes

\section{Demographics:}

Rose (Pink) - has been in Canada for 22 years

Had two childbirth experiences - both in Toronto

First child: 15-year-old son

Second child: 9-year-old daughter

Originally from Bangladesh

Born a Muslim

Wears loose-fitting cultural and Western clothing without hijab

Occupation: Childcare Provider

Sally (Yellow) - has been in Canada for 9 years

Had three childbirth experiences - first in Texas and rest in the GTA

First child: 12-year-old daughter

Second child: 7-year-old daughter

Third child: 5-year-old daughter

Convert to Islam due to marriage

Wears loose-fitting Western clothing with hijab

Occupation: Nursing Student

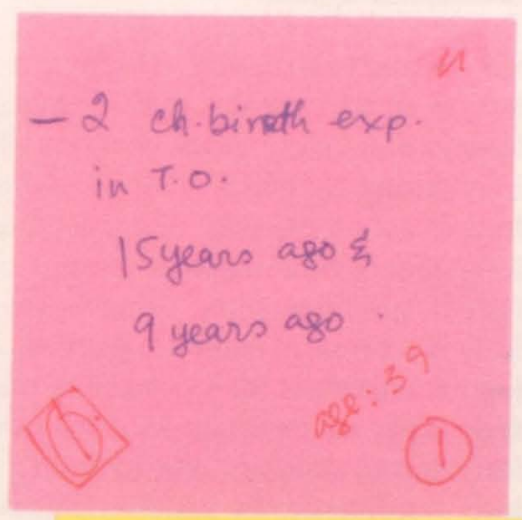

Sam (Green) - has been in Canada for 12 years

Had one childbirth experiences - in Toronto

One child 9-year-old son

Originally from Pakistan

Born a Muslim

Wears loose-fitting cultural and Western clothing without hijab

Occupation: Doctor

Mary (Orange) - has been in Canada for 9 years

Had two childbirth experiences - first in Hong Kong and second in Toronto

First child 10-year-old daughter

Second child: 8-year-old son

Originally from Bangladesh

Born a Muslim

Wears an abaya (long, loose-fitting dress) and hijab

Occupation: Stay-at-Home Mom

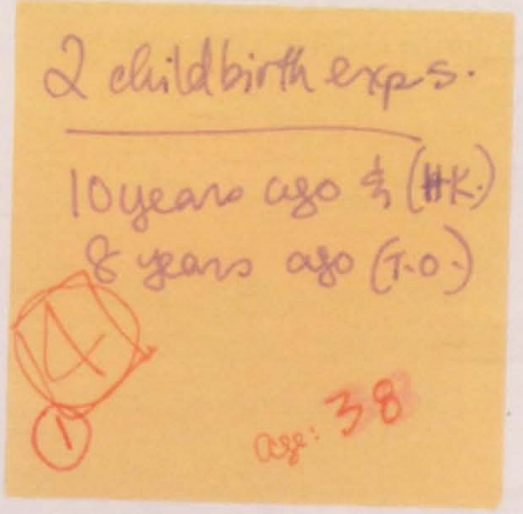




\section{Modesty (in general):}

Modesty in and expectation in Islam and is reflected in one's behaviour, speech, action, attitude and dress. Modesty is an attribute that is also expected of men, but in different ways

Modesty in dress requires the covering of the body in such a way that it does not attract undue attention.

Modesty during childbirth is especially important.

Modesty in action requires: doing good deeds and keeping away from bad deeds.

Modesty in behaviour requires treating others with respect and kindness.

Modesty in speech requires: using words that do not offend.

Modesty is a personal choice, regardless of religion

Modesty is important for non-Muslim men and women as well

Modesty is not an important issue for some Muslim women.

Modesty during childbirth means having minimal physical exposure to as few people as possible.

Modesty is influences by various cultural and societal factors.

Modesty is seen as protection.

Modesty is ingrained from childhood.

Intention is an important aspect of modesty

Three women are practicing Muslims from childhood. By practicing Muslims, I mean those who regularly and actively follow the basic guidelines according to Islam, as opposed to those who do not.

One woman is a convert to Islam from Christianity as a result of marriage.

Three women consider modesty to be first a personal choice, then a religious one. Of these three- two women feel this way because they grew up in societies that place a high value on modesty and the third woman feels it because she chose to convert and adopt the religion.

One woman draws attention to the fact that modesty is an issue for many non-Muslim women and not so big of an issue with some Muslim women as well

One woman considers modesty to be first a religious choice, then a personal one. However, it is important to note that she is also the only participant who used to wear a hijab prior to marriage, but took it off postmarriage because her husband wanted her to.

All four women consider modesty to go far beyond dress

Only one woman points out that modesty is also expected of men, albeit in different ways.

One participant states that modesty is a common feature of other religions as well.

From her experience as a doctor, one participant notes that women are generally more concerned about their modesty during childbirth than their husbands.
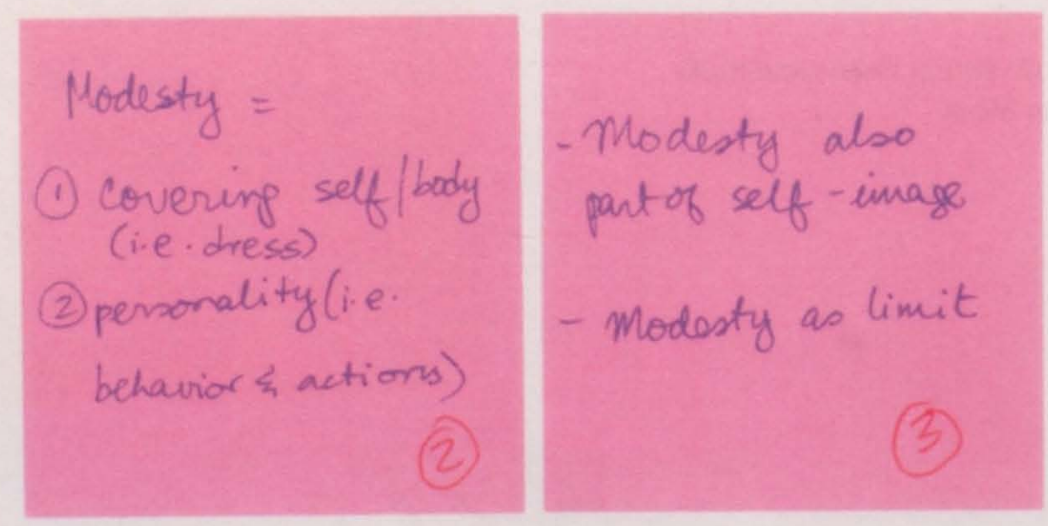
Modesty for Mustim ?:

Indres: cover all except face \& hando exp in front of men Inaction: no plysicical contact $w /$ men

Modesty in

attitude is

equally inp.

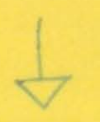

Behawior \& Speech

in Action.

(as stated or outlined in Quran)

- Concern for modesty 10 years ago was leso than it is now - increased sensintivity result of study of Istam
- Modesty in speech as well - demons trated by PAR. (48) - using termo like "private parto" $\therefore$ comfratable is noeded pain relief.

- Notion of Intention very imp. in Islam. (i.e. if we want to do the right thing but can't due to circumstances - its ok) (9)

Modeoty in Speech

Co using terms like "body parts" or 10 "private pants" i 0 wore accunatelanatomical terminolosy

However, PAR feels concern for. modesty is more rooted in (18) oulture/upbringing tham in relision. modesty $=$

covering not only from 77 but aleo w/ q.

(4)
Slamis View Modesty in dress, behavior as well.

Differences in modeoty of 5 is? exist - but (7) universal attributes of modesty includes kindress \& respect for Wutcess a rese others

cultural so societal Influences

(1) Nodesty as shame

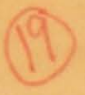


(2) Modecty as personal choice

\section{(2)}

To cover \&not wear revealing clothes is part of the cultural nom / ideal

(23)

Modesty is an

iosue for many Chistian $q$ as well

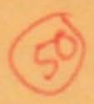

Modesty for Musclim q:

- imp.

- modesty during c.b.

even more imp.
(3) Modeaty as protection

\section{(2)}

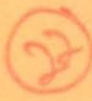

* When it come to modesty:

- Relision is secondang.

- Culture is primany.

\section{(24)}

Claim: Out Nuslim

t would feel

Same way

(70)

Relates Modesty

bark to neligion
PAR started to wean hijoab after 1st c.b. exp

Modesty as

imp. in daily liveo

in dress / belavior |

aetion etc.

(3)

\section{Babance}

Madesty in dress should make one contident. 
Modesty goee far far beyond doro-

\section{(13)}

differences in q's perronal preferences exist.

\section{So - accomodation} of sperial requesto can be manaed.

\section{(5)}

Motesty is :

a personal choice

q concerned about covering wrould do so nesandleso of relision \& viceversa
Acc- to Istam \& geneal society in most places:

modesty requires all body parts that are unaceentable to show in problic to be covered.

\section{(18)}

tmportant: Modesty is an ismo for non. muslime also: modesty is not an issue for some mudin? 9 .

Istam has rules but recognizes that prionities can shift incase of unforseem ciranstares

\section{(6)}

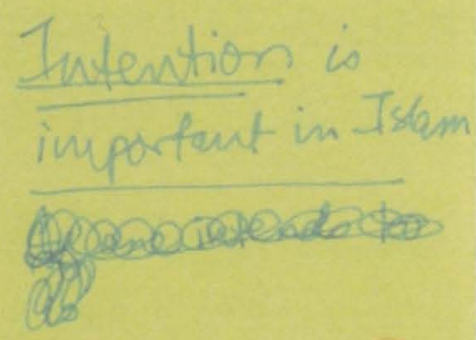

(61)
Non Muslim if also have this need to "cover" spectevy nimimal expooune to as ew peopleas possible - vs.

pustiple fmly. members peresent sh filuming kne bentts of a child. 


\section{Modesty (in dress):}

Modesty in dress means requires that women cover their bodies with loose-fitting clothing that does not show the shape of the body

Covering the hair is recommended in Islam, but it is not a requirement

Wearing a hijab for most women is a choice and it is not forced upon them

Dressing modestly warrants respect.

There are various ways of covering that are adopted by Muslim women. They include.

-loose fitting Western clothing with or without hijab

-loose fitting cultural clothing with or without hijab

-abaya with hijab

-abaya with niqaab (face covered but not the eyes)

-burqa (full face covered, with a mesh over the eyes)

Of the four women:

-one wears hijab and abaya

-one wears hijab with loose-fitting Western clothing

-two wear loose fitting cultural, as well as Western clothing without hijab

One woman states that in her experience she finds she receives more respect from people when she wears loose-fitting cultural clothing versus loose fitting Western clothing.

One woman states that women should dress in a way that they are comfortable without exposing any parts of the body which are considered universally unacceptable to expose in most societies. She also believes that dress should not attract attention because of either what they are or what they are not wearing. The objective is to blend-in

Women who wear hijab are not oppressed. They are active, contributing members of society who freely pursue and manage education, career as well as family.

Two women believe that modesty in dress is instilled from childhood through our previous generations.

The participant who is a convert to Islam chose to put on the hijab after learning about the religion and doing her own research. She read that paradise (heaven) will be more easily attainable by women who cover.
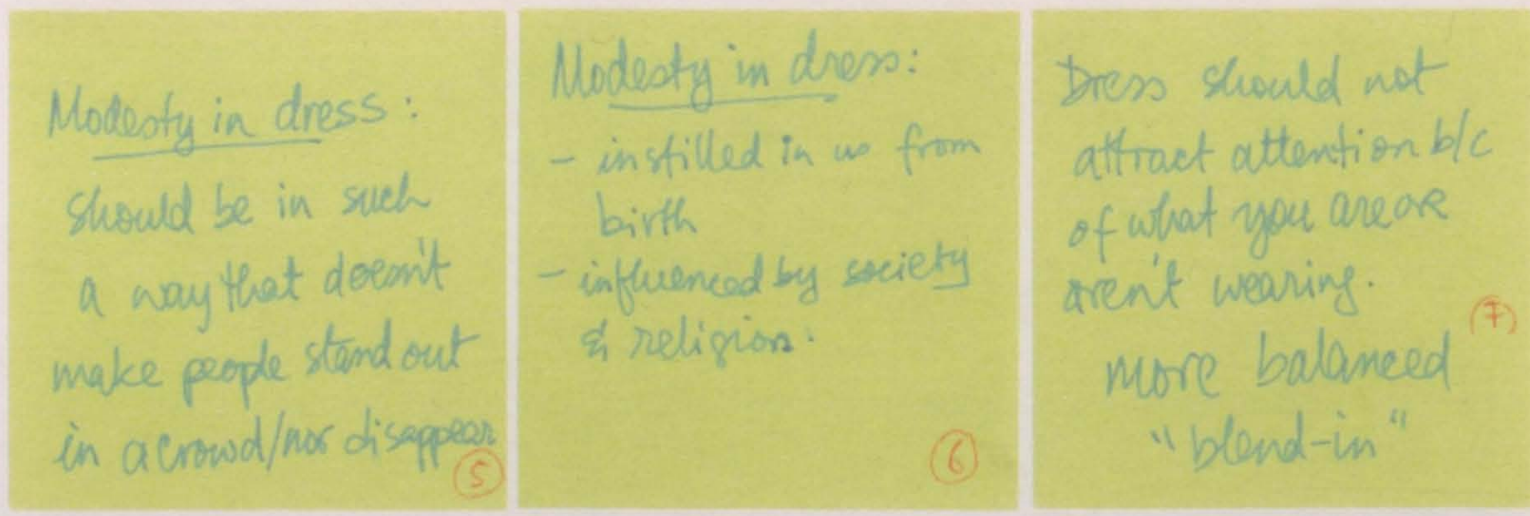


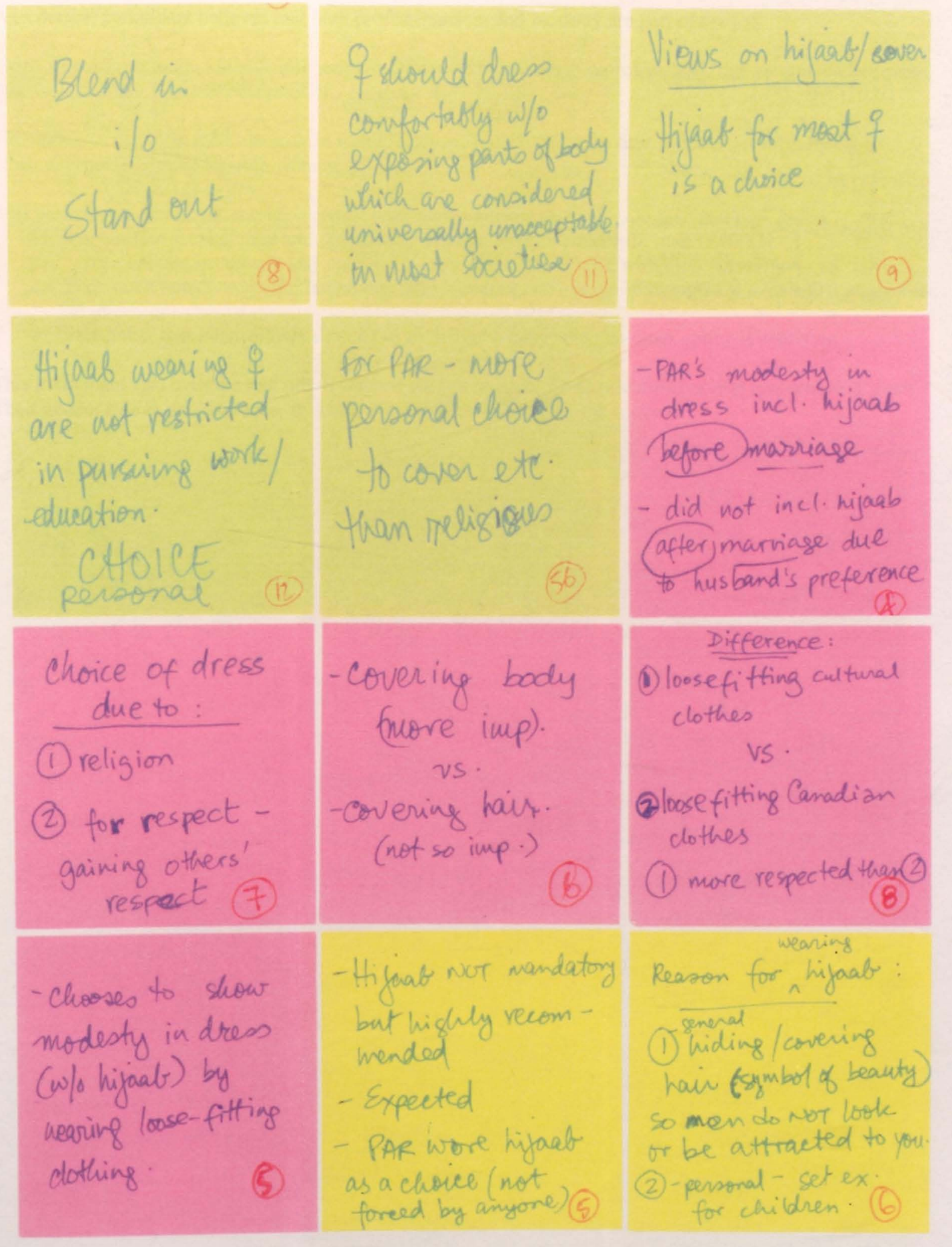

82 
- Motivated to wear hijab $b / c$ of quote in a book about paradise/ haven will be more easily attainable for 7 who comer
Hijab abaca

(2)

Various types of cover:

(1) only hifoalo

(2) hijab \& mboya

(3) loose -fitting clothes

(4) full Gurkha (5)
Modesty -

mainly focused on dress

(3)

Appropriate dress:

oneattidy

y cover body (alana)

u cover hair (hijab)

(Q)

83 


\section{Modesty (a female, Muslim doctor's perspective):}

As a doctor, participant believes that both professionalism and modesty are part of her job.

Professionalism often conflicts with modesty. Difficult choice, but as a professional, this participant believes that professionalism has to be a priority.

The ability to maintain their modesty in a childbirth situation is often the first concern put forth by most women, especially most Muslim women.

This participants advocates that any woman with such concerns should:

a) communicate (as clearly and specifically as possible)their need with their OBGYN

b) communicate (as clearly and specifically as possible) their need with their nurses

c) seek out a female midwife, but be mentally prepared that in case of emergency, a male OB may have to intervene

d) remember that Islam allows a male OB to deliver a baby when no other option is available.

This participant also believes that physicians are gender neutral. Their priority is to help heal and save lives Their gender makes no difference. It is only their skill that should be the concern.

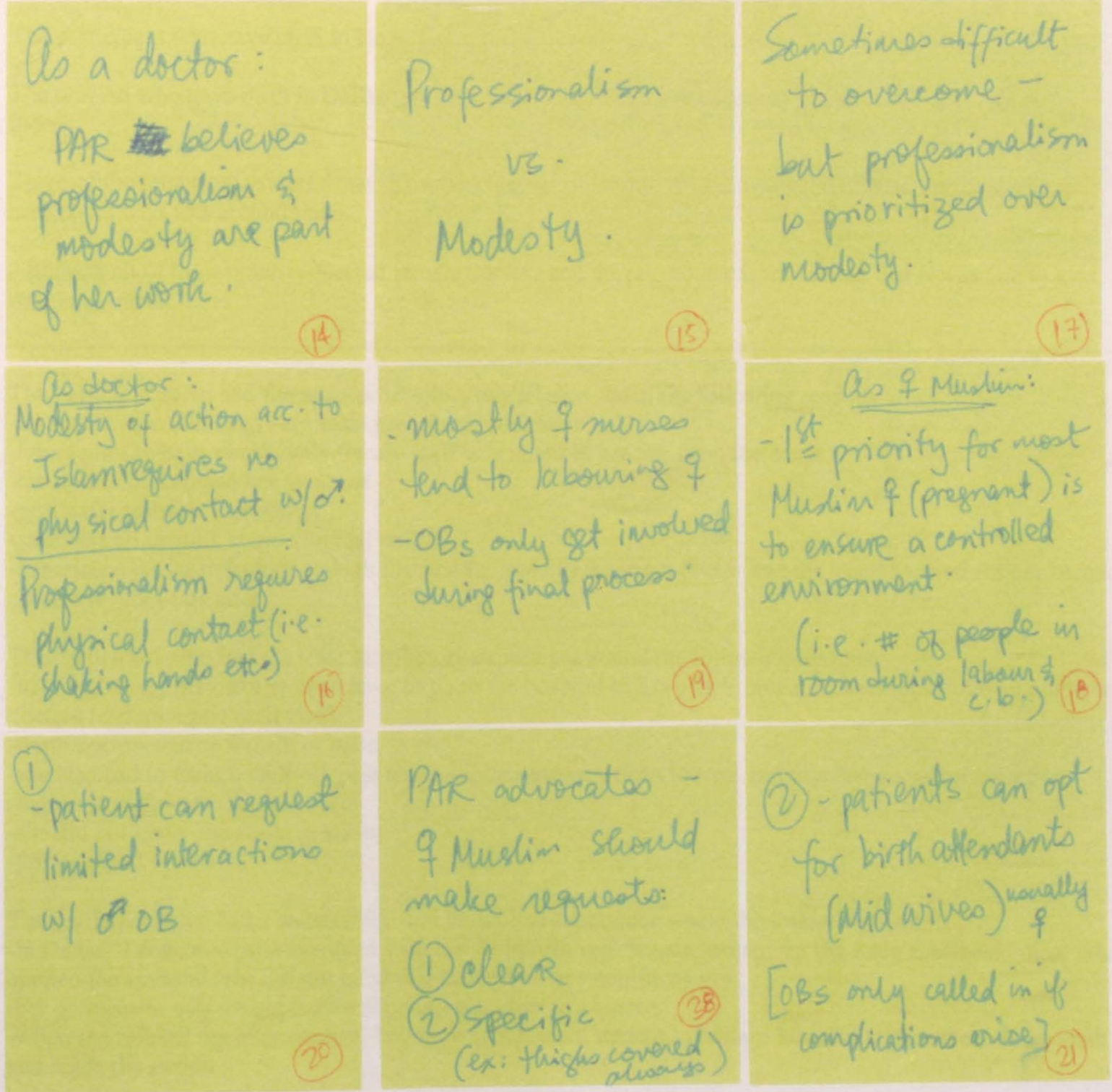


- Prionitizes Saving shelping requires human lise over

Neligious restrictions

(34) gender

neutrality

(32) from P.O.V - of Doctor:

- for $q$ who highly prioritize modestyPAR would recommend:

(1) comvinication

(a) $\omega / O B$

(b) w/muraes

(88)

(3) in case all foils -

(4) Hoe maidurives that Ielam allows but prepaned if OB ToBs to deliver the to intewere for babries wean no other emergeney. sption is owailable.

(59)

(6)

85 


\section{First childbirth experiences ( 4 women):}

Two of the women had their first childbirth experiences in the Greater Toronto Area (one 9 years ago and other 15 years ago).

One woman gave birth to her first child in a Hong Kong; 10 years ago.

One woman gave birth to her first child in a hospital in the United States, 12 years ago.

All births took place in hospital settings.

Three women had at least one of their childbirth experiences in Toronto soon after their arrival in Canada as new immigrants. One of the four women had been in Canada for over five years before she gave birth.

One woman had a highly satisfactory experience in Toronto (for pregnancy, labour, childbirth and post natal care)

One woman reported to have a satisfactory experience in Toronto (for pregnancy, labour childbirth and post natal care)

The participant who gave birth in Hong Kong had a very negative experience.

The woman who gave birth in Dallas reported that she also had a satisfactory experience there.

Three of the four women stated that in the final stages of labour, the pain was too intense for them to even care about who was around them.

Although all of the women requested female doctors and nurses, none of them stated that it was due to a religious reason

The participant who had the most satisfactory experience stated the following reasons:

-all female operation and zero male presence throughout

-her request to have exclusively female staff was noted in her file from the onset

-her doctor guaranteed her presence

-post natal assistance provided

-staff was all helpful, respectful and polite

-experience was satisfactory enough that she returned to the same doctor and the same hospital for her second childbirth, six years later

The participant who had the least satisfactory experience stated the following reasons:

-in Hong Kong, pregnant women have to go to the hospital to have their prenatal check-ups done by multiple doctors (different one each visit)

-these doctors can be female or male

-she also had to endure multiple post natal, vaginal examinations to treat and monitor a vaginal infection

-highly stressful time for her

-she did not know she could speak-up

-felt helpless

The participant who had a satisfactory first childbirth experience stated the following reasons:

-in Dallas, Texas, everyone involved with her childbirth was female, except for the male anesthesiologist who applied the epidural (she did not mind this because it was non-invasive)

-her permission was sought before allowing a student to observe

-when she refused the male student, but allowed a female student to replace him, the staff respected her wish and made the switch 
-nurses were culturally and religiously sensitive

-a midwife (on duty at the hospital) saw her through her whole childbirth

The second participant who had a satisfactory first childbirth experience stated the following reasons:

-had a female OBGYN who ensured her presence at the birth, but due to another emergency, the doctor had to leave and a male OB delivered the baby instead

-although uncomfortable with this change, the participant understood the situation and appreciated the fact that the duration of the delivery was short

-her satisfaction level was heightened because the nurses lived up to her expectations (they kept her covered much as possible, for as long as possible, and even after the delivery of the placenta, the nurses cleaned and covered her up as soon as possible)

\begin{tabular}{|c|c|c|}
\hline $\begin{array}{l}\text { PREGNANGY } \\
\text { - Is thing mentioned } \\
\text { to family doctor ( } 7 \text { ) } \\
\text { is need or preference } \\
\text { for } q \text { os }\end{array}$ & $\begin{array}{l}\text { Successful - divected } \\
\text { to wec where } \\
\text { majoity staff are of }\end{array}$ & $\begin{array}{l}\text { - toB validated PAR"s } \\
\text { request by making } \\
\text { a "Special note" } \\
\text { Validation built } \frac{\text { trust }}{\text { III }}\end{array}$ \\
\hline $\begin{array}{l}\text { Timportance of only } \\
O B \text { due to: } \\
\text { (1) Religion } \\
\text { (2) Personal Choice. } \\
\text { to Cover }\end{array}$ & $\begin{array}{l}\text { PAR's welfare (i.e. } \\
\text { mental health) given } \\
\text { importance. } \\
\text { "guaranteced" } \\
\text { omale presence }\end{array}$ & $\begin{array}{l}\text { - Keeping of a promise } \\
\text { to ensure no male } \\
\text { presence }\end{array}$ \\
\hline $\begin{array}{l}\text { familiarity w/ } \\
\text { staff (they know ne') } \\
\text { - ongoing relation - } \\
\text { ship formo bonds } \\
\text { builds trist. (3) }\end{array}$ & $\begin{array}{l}\text { - very content w/ } \\
\text { experience - } \\
\text { still keop in touch }\end{array}$ & $\begin{array}{l}\text { - Natural birth. } \\
\text { more exposure } \\
\text { - } 5 \text { days stay } \\
\text { exest postratal) }\end{array}$ \\
\hline
\end{tabular}


18t time $q$ - needed lessons on infant care.

(18)

Hst cob. exp in Dallas

"lucky" b/c all q except of anas the sislegist for epidural PAR was ok with it blconly back was exposed.

(10) Acc. to PAB

Permission had to be sought $b / c$ :

(1) student was not pant of the process so by law - needed permission.

(2) nijoar indicated par's focus on modesty (B)

- Right of birthing

q - valued important.

(16)

- Nurser were culturally sensitive.
- Praise for staff bic they:

(1) helped her

(2) were politer nice.

(19)

- While in labour(active) male student brought in to ask for fermi-

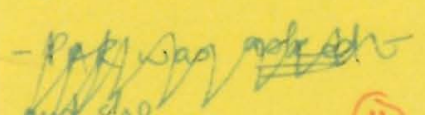

(11)

- PAR appreciated "seeing" the of student $i / 0$ being generalized as "a student"

(15) sion to observe

Exp. w/ 1 st childbirth satisfactory enough that PAR chose to return to same hosital - even though proximity was an issue (for $2=0$ cb.) (43)

- PAR was asked NoT told and when PAR refused they replaced student

- par was fine w/ that.

- HAPPY reval (12)

Respect for Religion

(14)

Nurse, \& Doctors response to mode ty:

- Respectful

- Given the chance to say" no"

(39)
- for $1 \frac{\text { st }}{3}$ delivery -

issues.

- midwives make a huge difference. q midwife on duty

$\therefore$ no

88 
PAR's cob. exp: years:

$\rightarrow$ new uni grant

$\therefore$ minimal exposure to edn-society.

$\rightarrow$ from Pakistan

Solonic nation

- Par was to not aware

that she had to a de for q OB. Livery conservative

In Pakistan -

labour \& delivery wand - all of

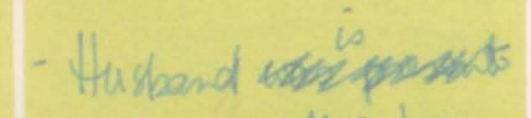
conservative by native

as is PAR.

(26)

(25)

Unforseen
Circumstance

neg: $O^{4} \partial B$ to

replace FOB

(28)

- PAR - dicospoornted

but understood

that 1009 OB

wo r that doing

his jots
Baby delivered by O $70 B$.

okay a bile:

(1) short duration.

(2) Emergency situation

(B) baby most in e (29)

Expectations of T.0. hosp.

(i) will to listen

(2) good nurses

(3) request to remain covered as long and as much as possible as respected. (36)
However - moalesty is also imp. To

observe around $q$.

(24)

Ensured that $O O B$ looked after PAR during pregnancy is also secured he presence for delivery.

(27)

Vaginal birth requires more exposure \& increases valera bility of birthing o.

(4) post placenta immediately covered q eared

Minimizing Exposure (37)

89 


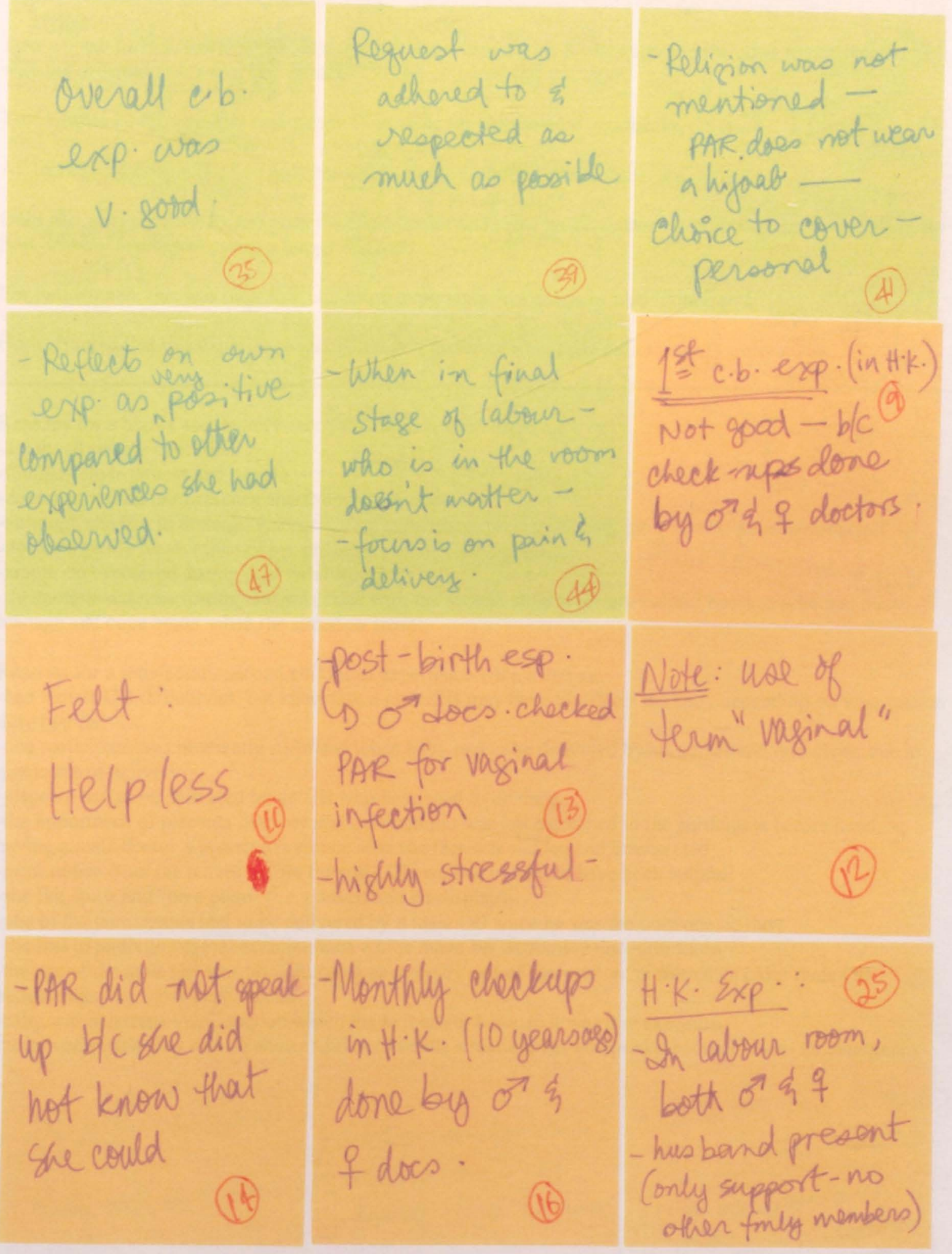


(2) In final stases natural $c \cdot b$.

$\therefore$ more exposure

\section{PAR very upser} Exposure of

"private parts" (29) \& closeness of ooc. to "private parts" very uncomfor tabde
In final stages
of labour - modesty
as covering is secondany - fous on dealing w/ pain is primany.

\section{(27)}

PAR didn't requse or request other wise b/c she thought it was not allowed. 


\section{Second Childbirth Experience ( 3 women):}

For one woman, her overall second childbirth experience occurred nine years ago and was highly satisfactory

Two women had satisfactory second childbirth experiences (one 8 years ago and the other seven years ago) with the exception of one or two issues.

Two women had to undergo emergency $\mathrm{C}$-sections for their second childbirths.

All three births took place in a hospital setting in the Greater Toronto Area.

Only one woman chose to stay with the same doctor and same hospital, even though she had moved since her first delivery and had to travel a longer distance.

For two women, this was their first childbirth experience in Canada as new immigrants.

Both reported that this experience was better and preferred over their out of Canada experiences.

Reasons for a highly satisfactory second childbirth were listed as:

-again, all female operation

-familiar environment and people

-doctor promised her presence and followed through

-although she had to undergo emergency surgery, she appreciated being informed by the doctor herself

-doctor's gentle reassurance of her presence and of God's presence

-ample care received during post natal stay

-the motion-sickness testing that was done with the second child was very helpful because it helped her to manage and cope better when the situation arose

Reasons for a satisfactory second childbirth experience were listed as

-had female $\mathrm{OB}$ throughout, but knew that a male $\mathrm{OB}$ may have to deliver the baby depending on who was on duty that day

-one participant was pleasantly surprised when baby had to be delivered by all nurses and midwives, due to quickness of delivery

-placenta had to be delivered by an OB who happened to be male

-the importance of placenta delivery and examination was not explained to the participant before hand, so having a male doctor's invasive presence after the fact was sudden and unexpected -prior notice from the nurses before the male OB walked in, would have been helpful -she felt stuck and "on a plate" (i.e. cooked and on-display)

-one of the participants had to be delivered by a male OB since he was the only one on duty

-she had to undergo vaginal examinations which made her extremely uncomfortable

-the age of the male $\mathrm{OB}$ was brought up as an issue by one of the women; preferred an older male $\mathrm{OB}$ because they are more experienced

-baby was delivered via C-section which made it easier because it was less exposure

-both women felt they had no choice, did not like the situation, but accepted it because it was an emergency
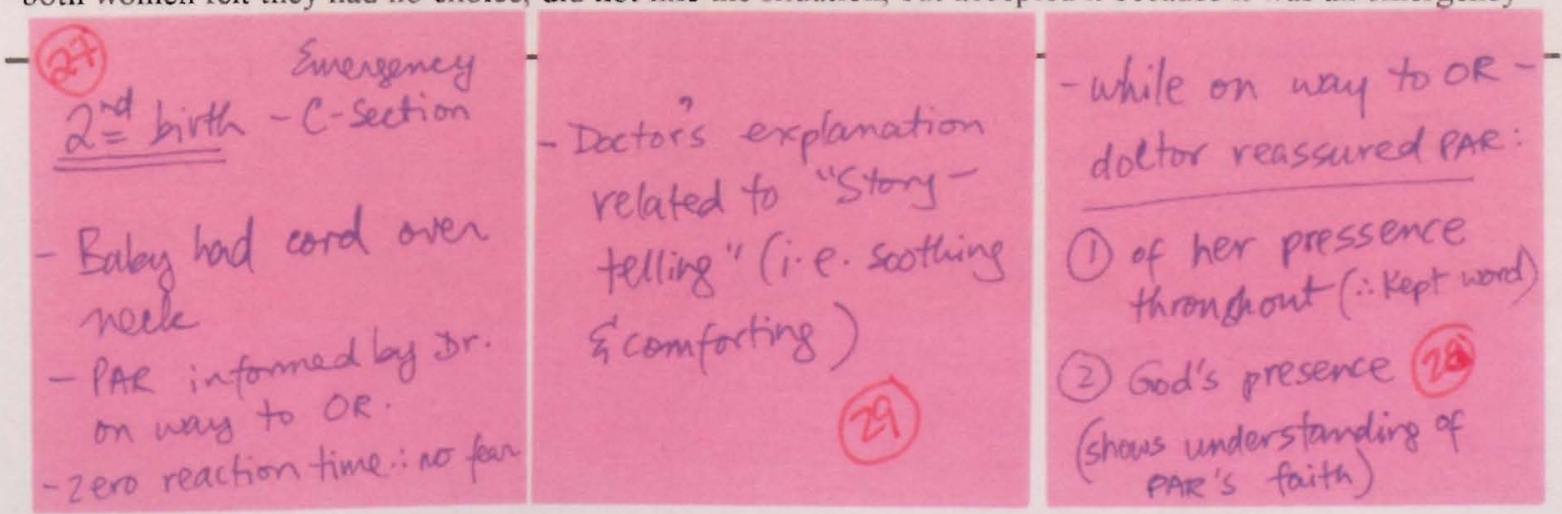
Positives about exp:

(1) kept their word (respected her values)

(2) provided ample post-natal care

\section{(39)}

- PAR was not infor wed before handso she was surprised \& felt" s tuck"

"on a plate"

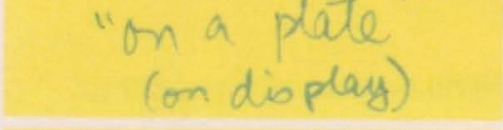
(on dis play)

Expectation for murses to askwas not met.

- Suddenness was upet. - ing (20)

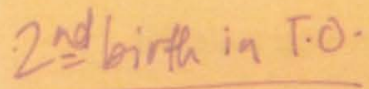

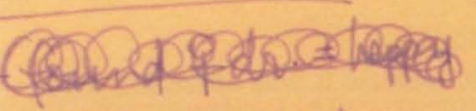
- new imigrant

- weaning hifaat
FAMILIARITY

enhanced

$2 \cong c \cdot b \cdot e x p$.

$2 \stackrel{n d}{=} c \cdot b \cdot \exp \cdot$ in GTA

COBGYN was q (17) Dprepaned before hand of porribility of $\sigma^{\circ} \mathrm{OB}$ delivering baby - So

PAR was okay.

$$
\text { ACC. to Islam: }
$$

Exanimation by

$\checkmark$ doctor allowed

incomos wasker when:

(1) Emergerey situation

(2) No $q$ doctor available

(2)

- found $q$ doctor $\therefore$ very happy

- all checkups by qoB

- no suarantee of qdrs.on day delivery
(49) -

- Knowledse - or

a heado -up as such was bolptul.

Helped PAR prepare! cope \& manase better.

- But baly was delivered by $q$ murses a miduives.

- afterbirth - 0 doctor had to deliver placenta

(18)

Did not like it but acceptable b/c it was an emergency or requived.
Due to latenese of night no qOB available su par did not have a cholce 
- husbandó

matter to PAR

- all she wanted

was a $9 O B(39)$

age of $d r$ matters

- older $O B O^{7}$ proffered over younger $O B$ F (still uncomfortable)
PAR - prayed that

She delivered on would be there (3)

- Prayers ane heavily depended upona day when for

- examination by or $O B$ made her feel uncomfor. table

(3)

Comfortable w/ everything else

D) less exposana bile more covered

(39)

(40)

94 


\section{Third childbirth experience ( 1 woman):}

This was the only participant of the four who had a third childbirth experience

She described it as "frustrating" from the onset

Reasons for an unsatisfactory experience were listed as:

-inability to access a female OBGYN for prenatal care (due to unavailability), even though she had insisted upon it

-as a new immigrant participant did not have access to the right resources

-however, the male OBGYN was sensitive to her discomfort and ensured limited contact (her hijab may have also triggered this)

-although delivery was scheduled (because the baby was small), the participant received an overdose of the labour inducing drug

-the baby came out much faster than anticipated and had to be delivered by a nurse because the doctor could not reach in time

-participant was happy about this because she felt she had by-passed the male OBs involvement -however, the male OB came in to deliver the placenta (again)

-again she felt intruded upon and like a "goose or a chicken on a plate" on display

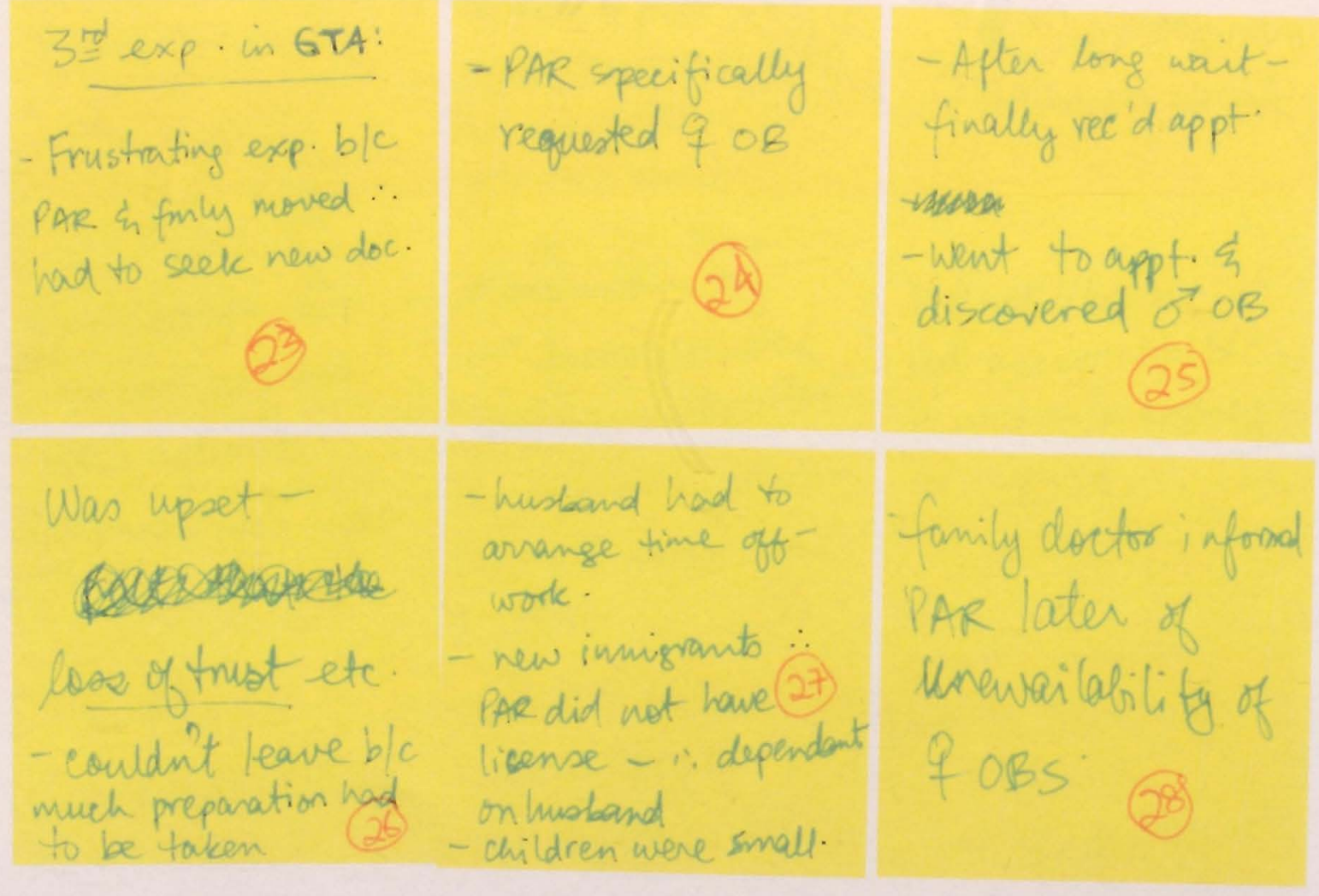


- PAR celt she - tried to find of should have

been told this before

- felt let-down

(29)

$\therefore$ demand for more GOBS exists

- Scheduled delivery blue 3 tr baby was $v$. small.

(32)

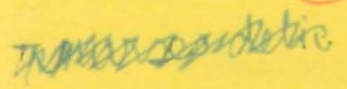

- PAR was overdosed on mede $\therefore$ increased contraction but $\sigma^{7}$ OB delivered placenta

- opturse had to deliver baby $b / c$ OB did not get there in time. (33)

- PAR relieved
- PAR felt $\sigma^{T} O B$ was sensitive to her discomforts limited contact as much as possible

(2) (31)

Respect)

. 


\section{Behaviour of Doctors and Nurses:}

One participant felt that her doctor and nurses not only treated her with respect, but also "cared"

This care she received throughout, for both childbirth experiences was exceptional

Two women felt they received respect from the doctors and nurses

One woman stated that she had mixed feelings about this issue because she experienced respect as well as disrespect

\section{Care received included:}

-an older nurse who ensured that the participants need to remain covered during labour was continuously monitored

-the nurse also spoke gently, held her hand and reassured her much like a mother would do -the participant believes that all nurses care, but this particular nurse went over and beyond her duty and provided care that was

-age of nurse was an important factor because she felt that older the nurses are the more experience they have to understand the needs of different women

-another nurse also took the time to sit by the new mother's bedside at night and intermittently her to access and hold her baby

-care such as this is part of one's intrinsic nature and not done because their profession demands it

\section{Respect received included:}

-nurses and doctors who limited the amount of examinations necessary because they felt the women's discomfort and noted their need for modesty

-nurses always spoke respectfully and tried their best to accommodate the women's requests and needs -for the most part, the nurses' treatment of one patient to another did not vary

Disrespect received included:

-two instances (one direct, one observed) of nurses who were rude to their post natal charges -direct-nurse refused to help the participant (who had just had a C-section) to reach her baby or wash the breast pump

-observed-another nurse shouting at another lady who had a language barrier

-weary of labeling it discrimination, but suspects it may have been
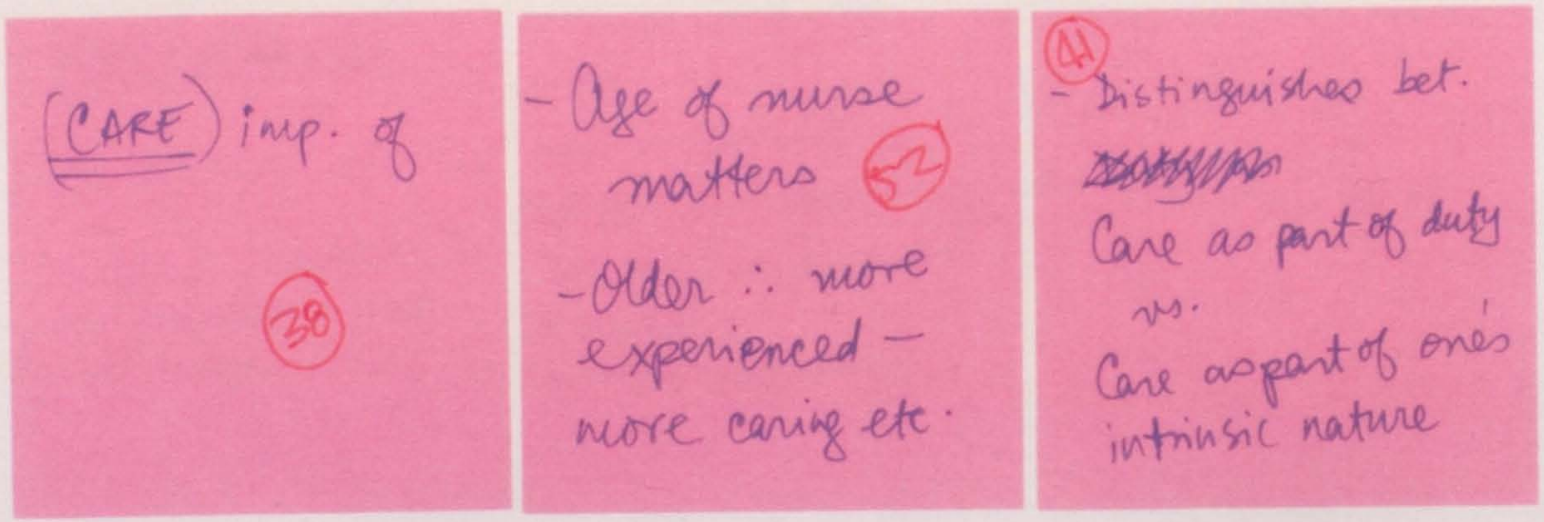
$2^{\text {nd }}=\exp$. of care received by nurse n perrisission to PAR to scream if needed.

- older

nurse as grandma

- on going existing at present. for 1 - $=$. b :

older nurse gave (Si) figure. (5A) rel. W/ nurse even

(bet. nurse/mom/son)

Older nurse =

as mom figure

- Ensured that PAR remained covered - truly "cared" (53)

- Feelings (CAFE

very imp. to PAR.

(5)

Nurse was informed of PAR's need for modesty sion by PAR's doctor.

(5)

* Care is simple just mean wells help patients.

* Care is simplebut concept runs deep.
Care exp. \#1

- nurse who sat w/ PAR for a while every night - helped PAR to hold her baby etc.

- Acc. to PAR: nurse showed care beyond "duty"

- nurses' mom -

like care - (50) attributed to her intrinsic nature Caring beyond "duty"

- Nurses were culturally competent \& respectful

(4)
- No discrimination exp. at all.
Nurses =

Respectful

$$
\text { of }
$$

(15) PAR's request all \& who regard modesty as an issue (regardlono of religion) need to communicate

98 


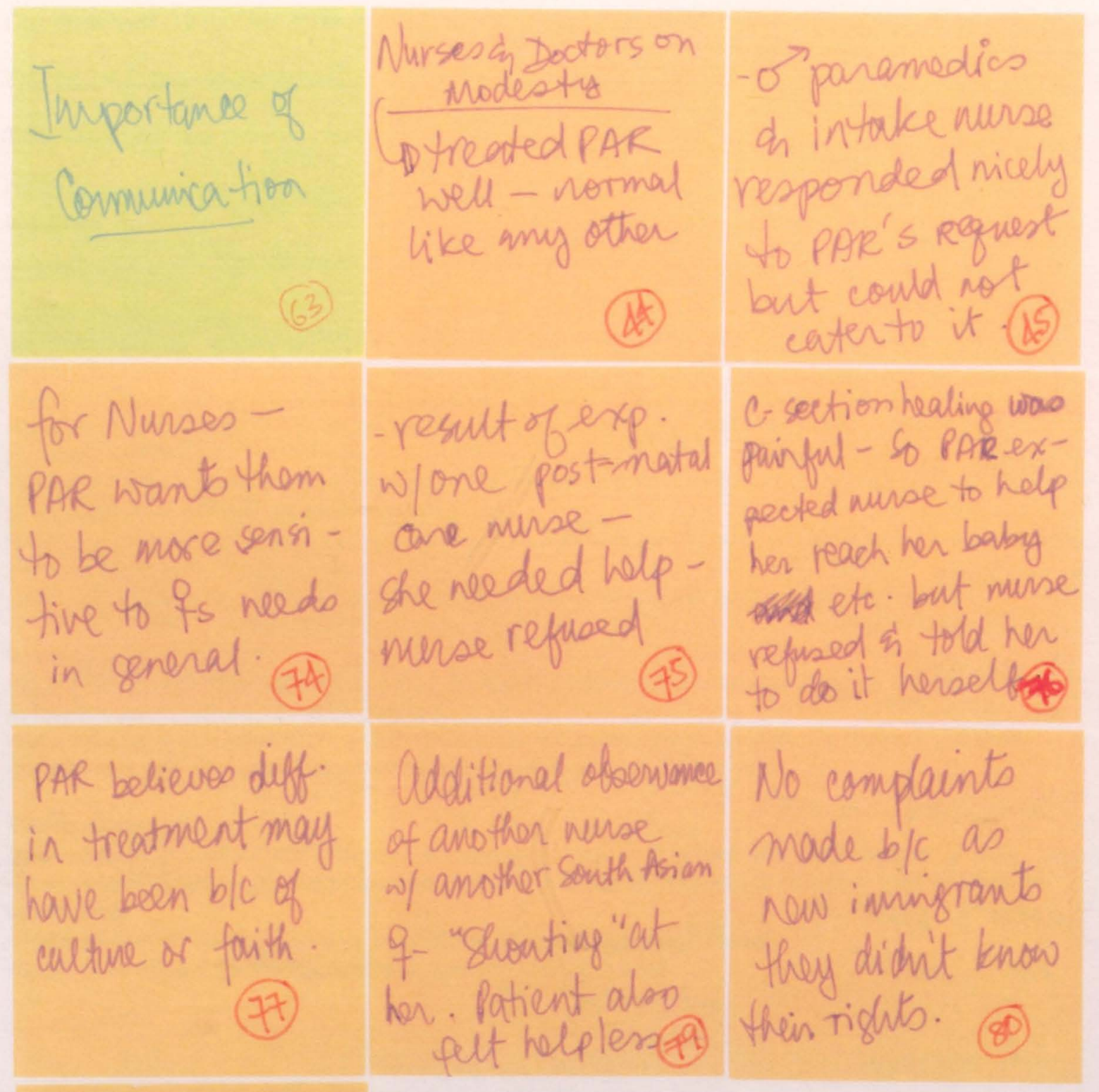

- Weary of labelling it "discrimina."

blc she doesn't know for sune

(48)

99 


\section{Expectations:}

Expectation to have an all female experience:

-was met for one participant fully for both of her childbirth experiences

-was mostly met for two women

-was met for one woman for one of her experiences, and not for the other (outside Canada)

Expectation to be covered and well-treated by staff.

-was met for three women for all of their experiences

-was met for one woman for one of her experiences, and not for the other (outside Canada)

Reason for expectation not being met (outside of Canada):

-in Hong Kong, women go to the hospital for regular check-ups during pregnancy and can be checked by whichever doctor is available (male and female)

-in Hong Kong, post natal monitoring of a vaginal infection was done by male doctors -participant could not state her needs because she did not know how, or even if it was allowed

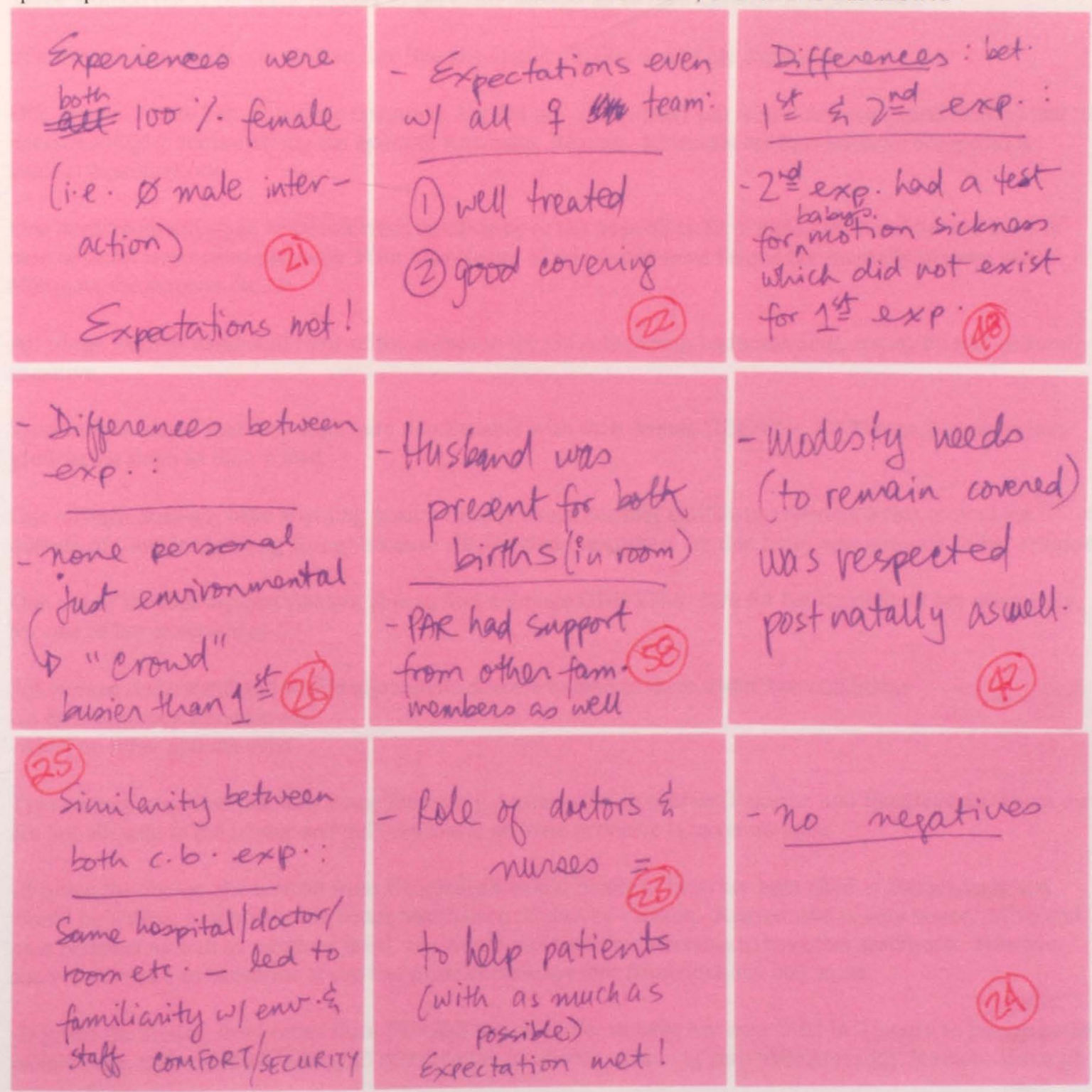


- Happy w/Experience

- No complaints
Negatives:

Ist birth - None (15y)

vod birth - oven - $(9 y)$ crourding led to ass lack of privacy.
- Care reíd was also afferted berause murse: patient ratio was increased (46) 


\section{$\underline{\text { Similarities and Differences: }}$}

For one woman, both of her childbirth experiences were very similar. Only one difference was stated.

Two women had one of their experiences outside of Canada, so each experience was different

One participant only had one child (not applicable)

For one woman "timing" made a huge difference between the three births. She preferred the final experience because it was the shortest (two hours). Reason: the shorter the labour, the shorter the exposure.

One woman also stated "lack of privacy" for the childbirth experience she had in Dallas, Texas where many labouring women shared one large room and were only taken to a private room when they were ready to deliver. Curtains separated each bed, but did not offer much privacy because they kept moving. In comparison, the privacy that is offered to labouring women in hospitals in the GTA was sincerely appreciated

Three women relied on silent prayers to get them through all of their childbirth experiences.

Intensity of pain made one woman feel like she would die (for one of her experiences)

One woman wanted an all female operation, but did not mind (then) and would not even mind (today) that the anesthesiologist administering the epidural was male. Reason: Epidurals are non-invasive compared to vaginal examinations.

One participant expected to be informed in advance of the possibility for a male OB to deliver the baby in case of unforeseen circumstances. Prior knowledge of this would have helped her mentally prepare and eliminate the surprise factor.

All of the women commend most of the nurses in the GTA for being understanding, respectful and culturally sensitive.

Two women stated that they were very comfortable with their female OBGYNs (in Toronto) because they grew to see them as their friend.

One woman does not have anything positive to say about her first childbirth experience that took place outside of Canada (in Hong Kong) because she felt that the country (at that time) was ignorant of her religion.

One out of the four women was not able to find a female OBGYN to care for her throughout her pregnancy, for one of her experiences.

All women understand and would allow male doctors to tend to them under two conditions: -in an emergency situation and

-when no other options exist

Two women specified that they come from very conservative countries (Pakistan and Bangladesh) where men are not allowed in the labour and delivery ward, so male presence is never an issue.

-If given the choice, the woman from Bangladesh would prefer to have her next child in Bangladesh, and would be willing to sacrifice the better health care offered by Canada. Reason: she is very conservative and on a personal as well as religious level, she would feel more comfortable to have this assurance. However, she would make an exception if she had prior knowledge that complications may arise.

-If given the choice, the woman from Pakistan would prefer to have her next child in Toronto to ensure better patient care, and would be willing to sacrifice the guarantee of having zero male presence. Reason: although 
she is conservative, she is also a doctor and as such is more open, understanding and realistic about setting her priorities.

Two women also state that a certain level of modesty should also be maintained even in the company of other women

Two women continuously praise and thank God when they recall their childbirth experiences.

From the perspective of a nursing student, one participant believes that although cultural sensitivity is expected and taught, it is not consistently and actively practiced. Reason: male students are sometimes assigned to hijab-wearing women, even though they know that the women will refuse or be uncomfortable.

None of the women used religion as support for the adherence to modesty. It was just stated as a personal choice.

Three women stated they would seek out and recruit female midwives for any future births. Reasoning offered by one woman: midwives are sensitive and soft, while doctors are usually clinical and cold

Two women mentioned that they have no intention of having more children. No reason was specified.

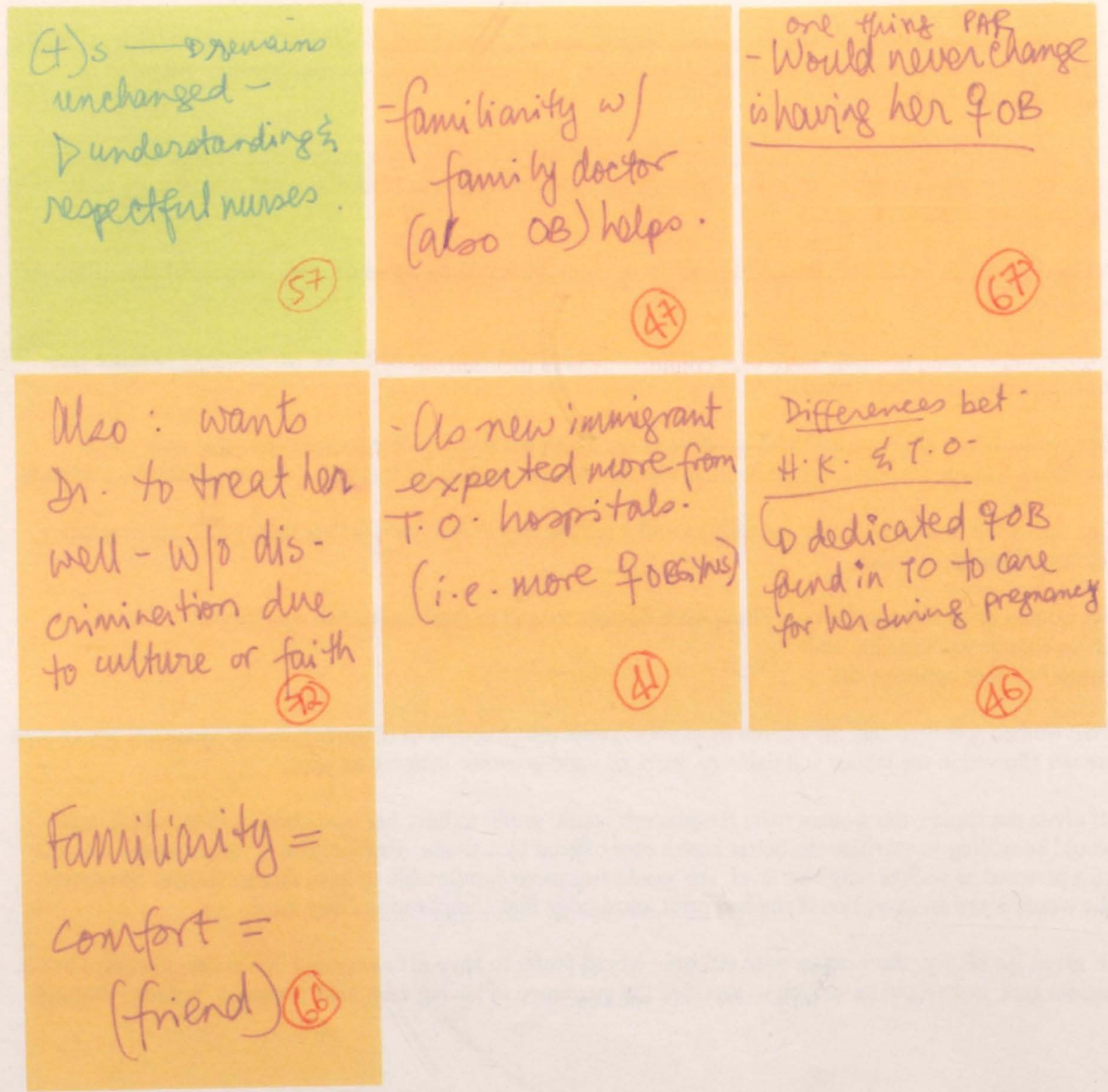


Diffs - in prenatal

Care provider in tH k-

VS. THO.

(15)

$H \cdot K \cdot \rightarrow$ hospital for cane

ti. $\rightarrow$ fomstiplo does. cones throrefout
(-) worst thing

07 issue in $H \cdot k$.

would never wont

to face again $(t)$ s about $c \cdot b$. :

$-\forall k: \rightarrow$ nothing

- T.0. D having q oB understood "of f-duty"

(15)

Differences bet. cob.

- more comfortable w) To Births bic hospitals in G.T A ot fer more privacy

(26) sol thanked due to comfortable (like a shortening of labour time

PRIVACY

- in Dallas pesrogeg when in labour -

bic patients get a room to themselves

(23)

Differences

$1=$ - all $q \therefore$ comfortable

- Male thasthesiolegist was okay bic procedure was nonimprove.

(At)

Non invasive vs. Invasive

$\therefore$ al ark of privacy b) c curtains fly open hare increased i chance of being seen by others.

$2^{n d}=13 \frac{r d}{=}-$

(49)

TOB seen as more invasive bic delivery of placenta required exposure of private parts"

104 


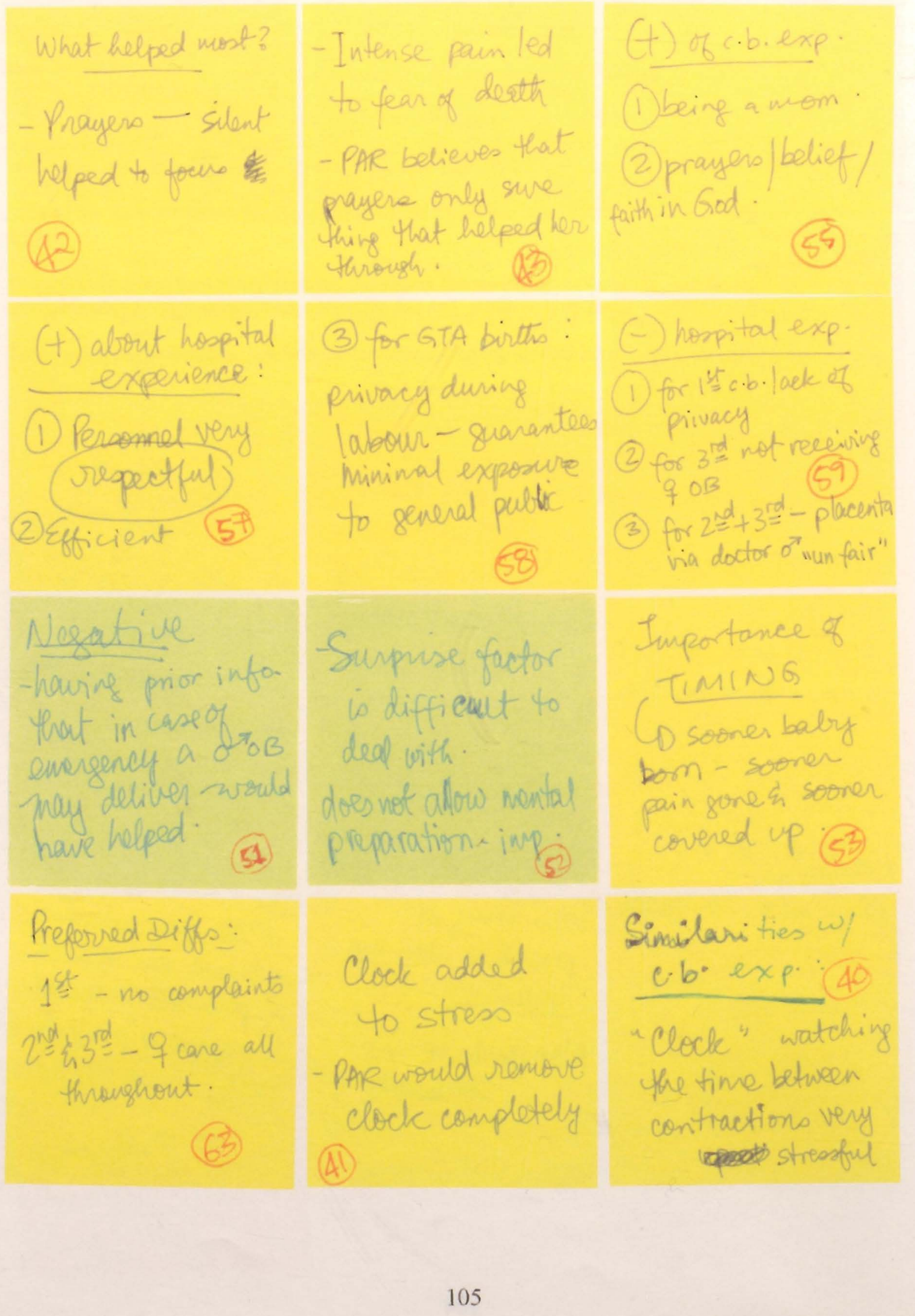




\section{Recommendations:}

Three of the women voluntarily offered recommendations for bettering the health care system's ability to cater to the needs of Muslim women (or any woman, for that matter) who value modesty.

All three women stated that more women need to pursue this specialization.

One woman (the nursing student) also suggested that the Ministry should offer more funding to make midwives readily available for pregnant women. She also recommended that more women need to be encouraged to pursue all specializations so they can be accessible to all women.

One woman also recommended that the city build more hospitals that cater exclusively to women and women's issues, staffed by women as well
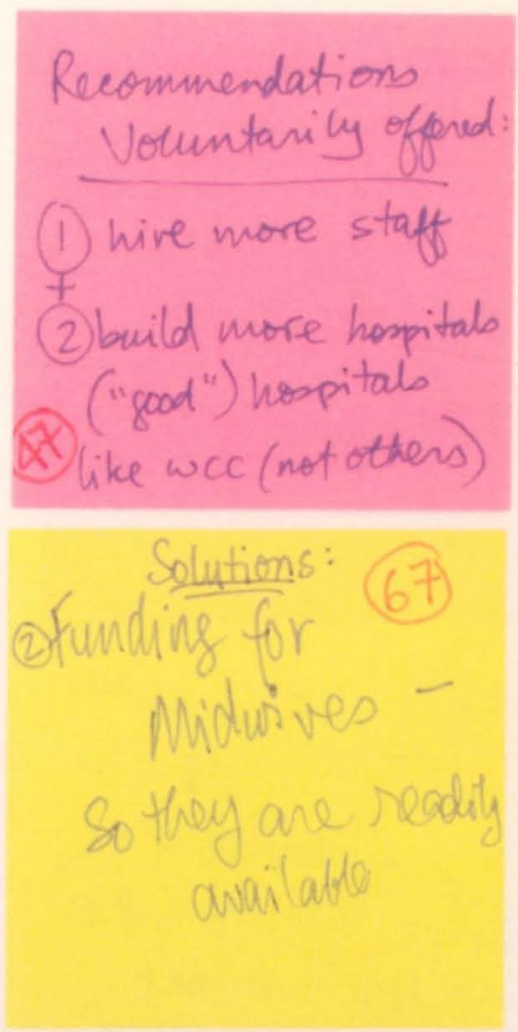

Pregant | Labowing : :

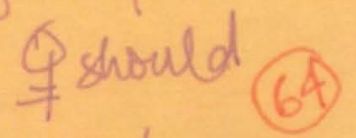

alusang howe a

CHOICE

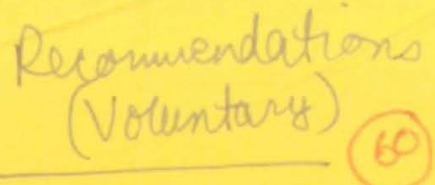

meed for more

Q in tis profersion

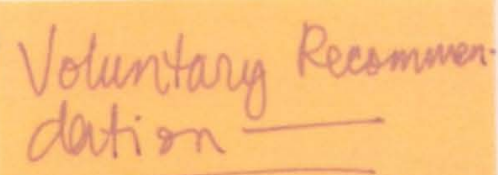

due to denumal for qOBs - wore q Should be encowlaged to punoue spealizan.

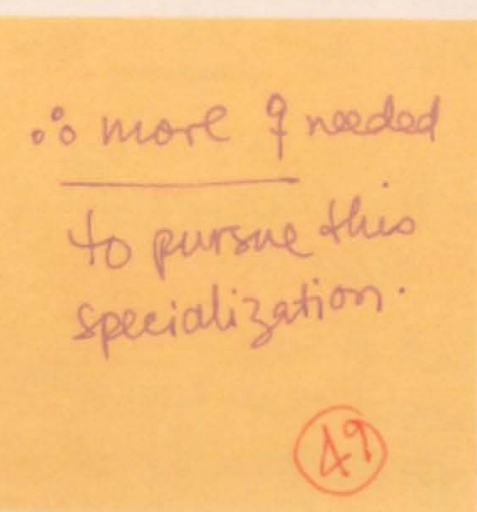

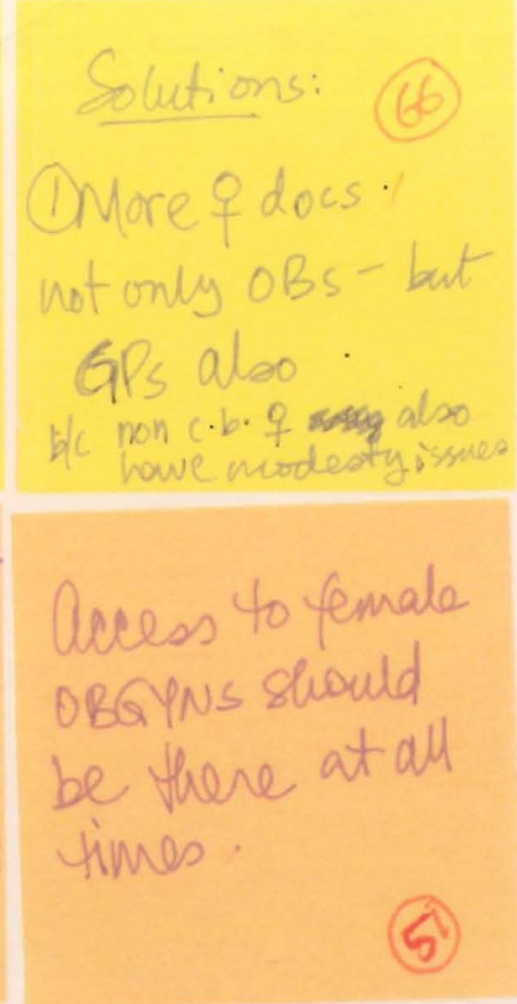

Accessibilify for

both $q+070$ obs

bc some q may

prefer of obs. 


\section{Ideal Childbirth Experience:}

One woman would not change anything about either of her childbirth experiences, because for her, they were ideal.

The rest of the women stated that they would all require exclusively female staff and that they would take more responsibility to seek out female OBGYNs or midwives. They would use their existing knowledge to their advantage by seeking out resources to aid them. They all wish to have more control.

One woman (the doctor) would take a professional and practical approach and present her $\mathrm{OB}$ with a birth plan that has the baby's safety and her own need for modesty to top the list. She would also be as specific as possible to ensure that she clearly communicates her needs to any one who will tend to her.

One woman stated that she would not mind traveling further to be able to access a female OBGYN if necessary.

Two women stated that as a final resort, in order to guarantee the presence of their female OB, they would schedule their delivery (be induced).

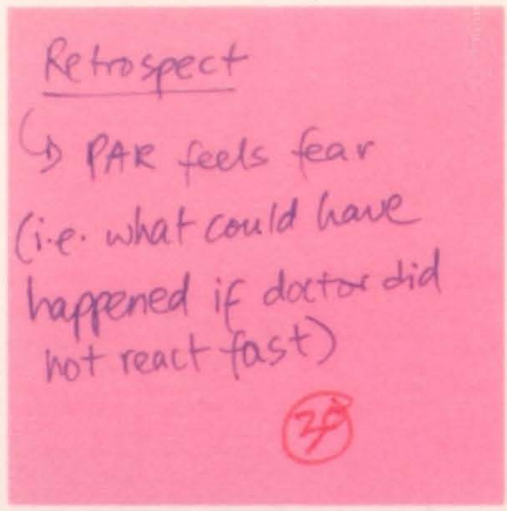

$$
\begin{aligned}
& \text { Team work } \\
& \text { important \& } \\
& \text { necescany - }
\end{aligned}
$$

- Murses / doctors s patients.

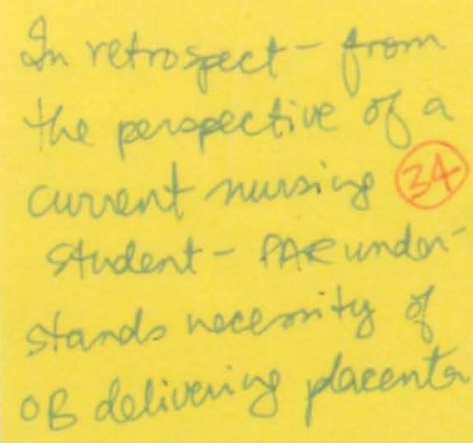

CReDIT for squing baby:

$$
\text { (1) to God }
$$$$
\text { (2) to doctor s team. }
$$
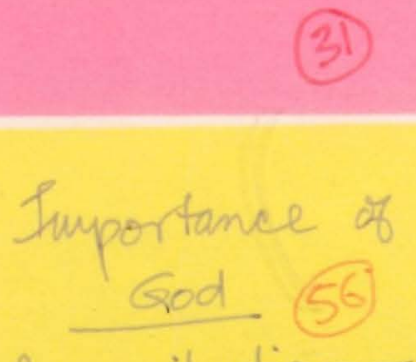

In all situation whon every thing else tails-exp. when no sthen way - God is there

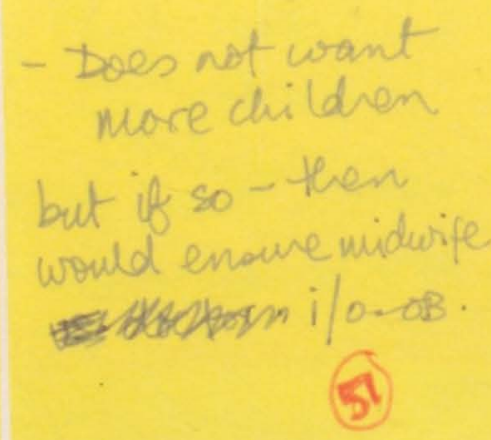

Whenever good health is spoken of God is remembered and mentioned

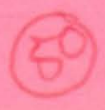

What difference would liele? * priar notice of unavau lability of $f O B$ So PAR woulda't have to be sumprised PAR and fomly new
to Canada $\therefore$ knowledere of resources was bivited 


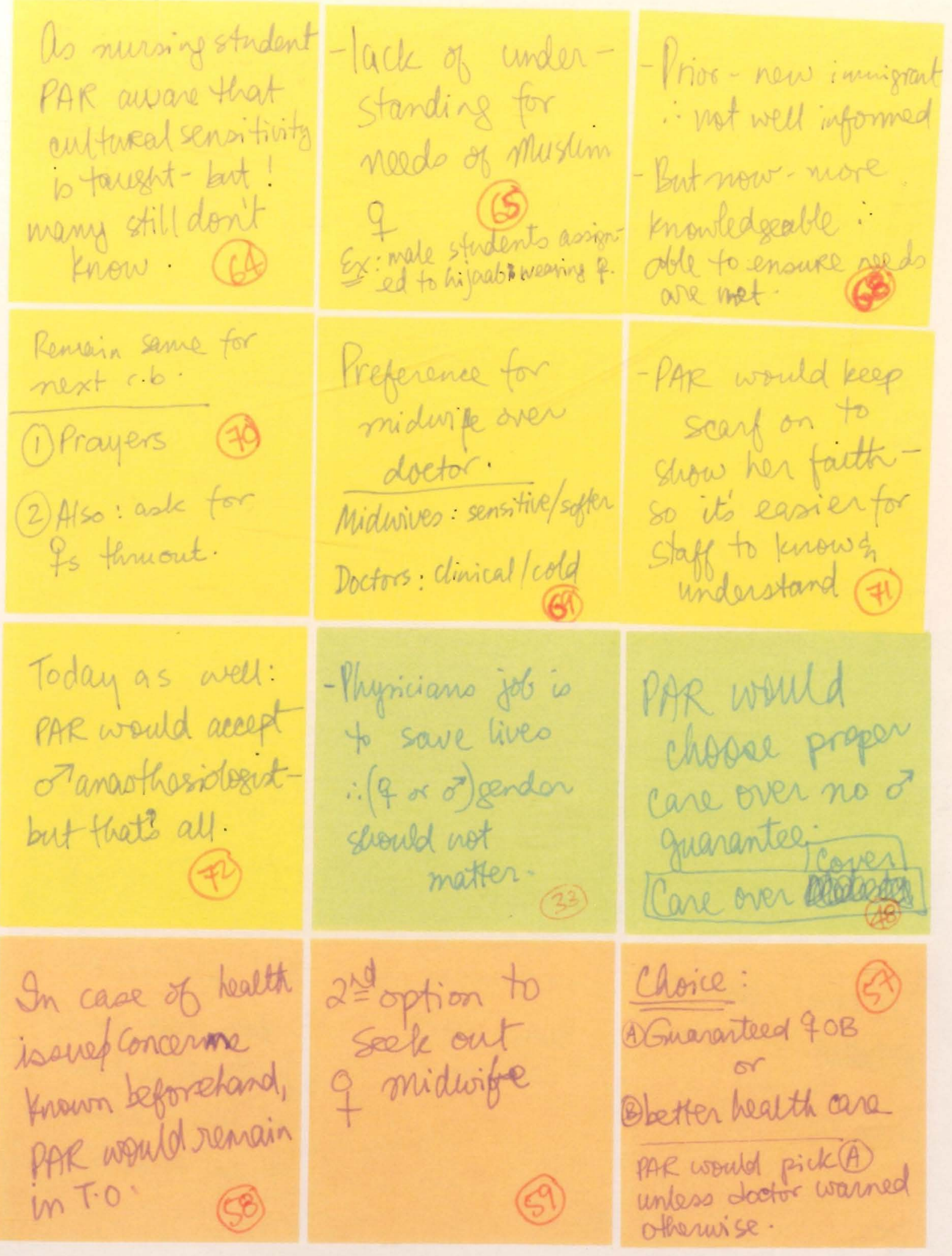

108 
Ideal $c \cdot b \cdot \exp$

(1) Ensure $a q \circ b$ (men)

To combat the (15) issue of "doctor -
on - duty" -

PAR would schedule the deliveny (to be induced) So $9 O B$ guarenteed thru out.

Specifics

(1) being covered amap.
alap:
a sap.

(2) legs covered thruout

(3) limited \# or people

(4) guarented qOB-mugbe coledule inductions

In home countryBangladoch, OBSS are all $q$

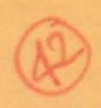

(2) I midwifeseek out by self.

(3) if neithen - then PAR would seek out 9 $O B$ funther away as travel is more accers-

All 7 a must!

(46) ible-kido groun

Next c.b-exp:

- Would prepares present bivth-plan to $q O B$

- Babiy' safety s
PAR's modesty wrould
top the list. (4)

Would Take a professional \& practical approach guaranter $9 O B$,
patient care would
be compromiseds
be of low number to
murses to eater topotiont. guaranter $9 O B$,
patient care would
be compromiseds
be of low number to
murses to eater topotiont. guarantel $q O B$,
patient care would
be compromiseds
be of low number to
murses to eater topatiens delluo. Paka stam

No intention of kowing more children
Comparison of falcistani hospitalo \& Can hospitalo. * PAR prefers Cdn - exp.
But if at all then PAR would prefer to go home to Bangladesh. b/c all OBGYNS are q. 
Guaranteed

q OBS

\section{(5)}

M temale operation!

in case of emers. par understando $\checkmark O B$ way have to intervere
Ideal pres. $/ c \cdot b$.

Scenanio

\#1 prionity is to secure $9 O B$ (gonante) throughout

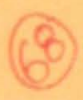

Ideally - PAR would prefer one qob to eave for her Yuruout - even for postnatal check ups.

\section{(6)}

If male $O B$ neecesory then PAR would main thing by one $O^{7} O B$ i ( B muttiple (as in H.K.) prefer to be soen that is okay b/c Islam allows this if:

(1) Emersency sit.

(2) No other option 


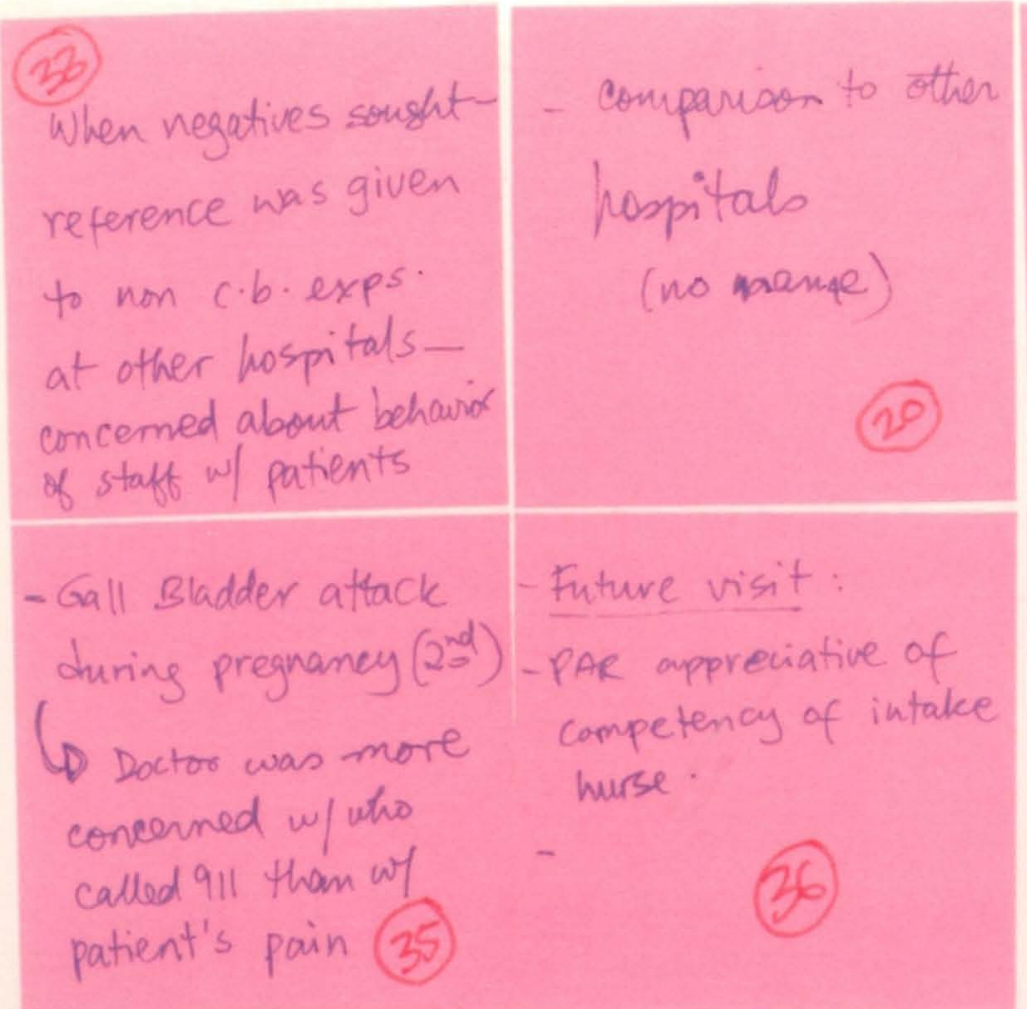
- General (won c.b.) hospital experience made her as patient feel:
(1) like aburden
(2) voiceless (3) incompetent

- Dactars us nurse - Questioning doctors competency/sensitivity

$\rightarrow$ f wavilly foens duning c.b.

$\rightarrow$ but hudbandó also underso streso worrying alrout well being of wite. (6)
Role of hudbando.

- monallas minimal interference

- PAR's Wurband conoenvative but entichtened : underetanding of stomet 


\section{APPENDIX D:}

\section{Tree Diagram}

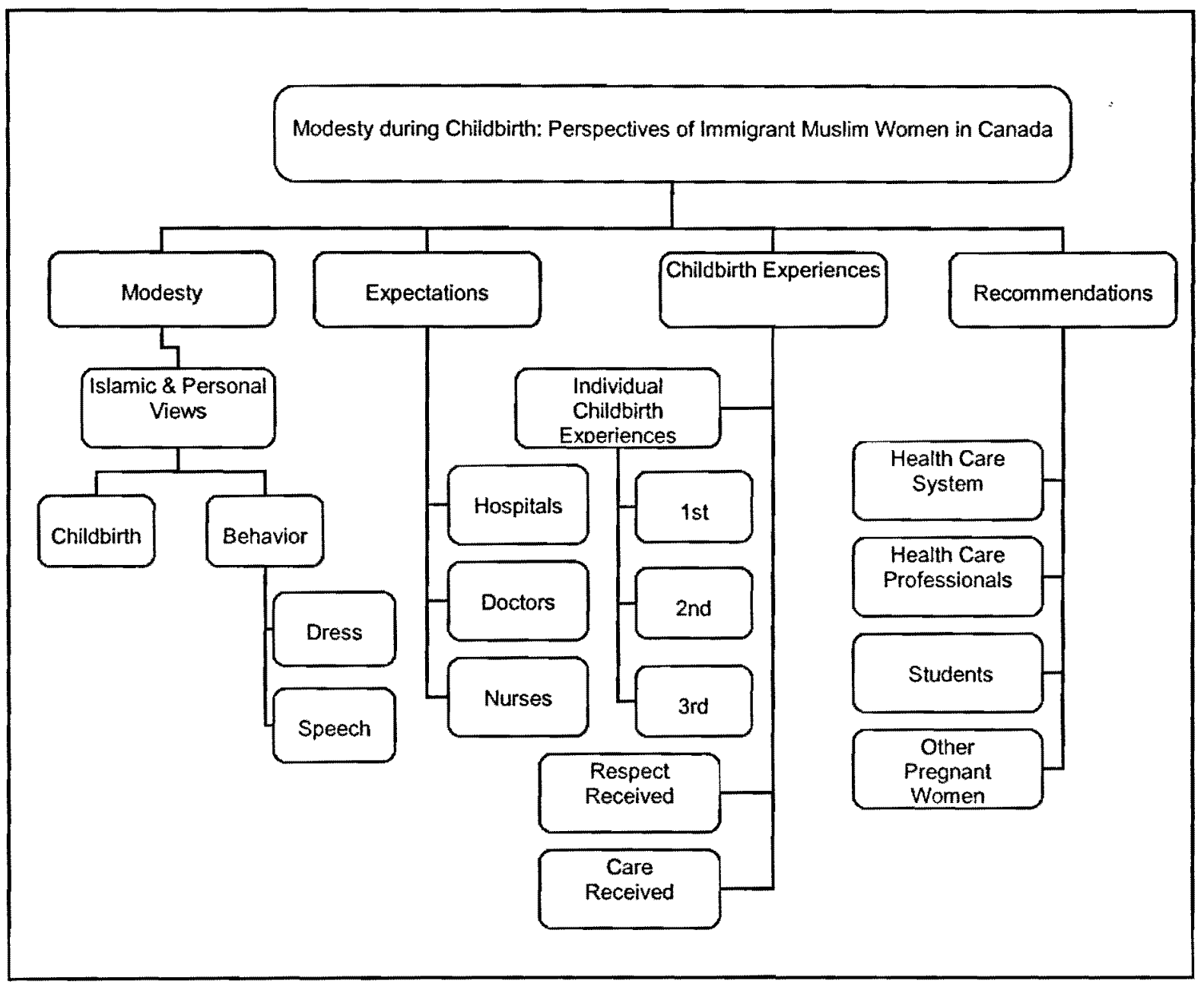




\section{REFERENCES}

Ali, A.Y. (1989). The Holy Qur'an, Text, Translation and Commentary. Maryland:

Islamic Propagation Centre International.

Arif, Z. (2006). Shame on you. Nursing Standard, 20(36), 32-33.

Bensing, J.M., Den Brink-Muinen, A.V.M., \& De Bakker, D.H. (1993). Medical Care, $31(3), 219-229$.

Ben-Ze'ev, A. (1993). The virtue of modesty. American Philosophical Quarterly, 30(3), 235-246.

Callister, L., \& Vega, R. (1998). Giving birth: Guatemalan women's voices. Journal of Obstetric, Gynecological, and Neonatal Nursing, 27(3), 289-295.

Callister, L. (2009). Mamawasi: Culturally sensitive birthing for Peruvian women. Global Health and Nursing, 14, 66.

Chalmers, B. \& Omer-Hashi, K. (2002). What Somali women say about giving birth in Canada. Journal of Reproductive and Infant Psychology, 20(4), 267-282.

Collins, E. (2008). Recognizing spirituality as a vital component in mental health care. In S. Guruge \& Collins, E. (Eds.). (2008). Working with immigrant women: Issues and strategies for mental health professionals (pp. 89-102). Toronto, ON: Centre for Addiction and Mental Health (CAMH).

Collins, E., \& Guruge, S. (2008). Theoretical perspectives and conceptual frameworks. In S. Guruge \& E. Collins (Eds.). (2008). Working with immigrant women: Issues and strategies for mental health professionals (pp.19-38). Toronto, ON: Centre for Addiction and Mental Health (CAMH). 
Condon, M.C. (2004). Women's health: An integrated approach to wellness and illness. New Jersey: Prentice Hall.

Considering a midwife? (2009).Midwife pregnancy information. Retrieved august 19, 2010 from http://www.pregnancy-info.net/midwives.html

Creswell, J.W. \& Miller, D. (2000). Determining validity in qualitative inquiry. Theory into Practice, 39(3), 124-130.

Creswell, J.W. (2007). Qualitative inquiry and research design: Choosing among five approaches. Thousand Oaks, CA: Sage Publications.

Creswell, J.W. (2009). Research design: Qualitative, quantitative, mixed methods approaches. Los Angeles, CA: Sage Publications.

Denzin, N.K. \& Lincoln, Y.S. (2005). The sage handbook of qualitative research. $3^{\text {rd }} \mathrm{ed}$. Thousand Oaks, CA: Sage Publications.

De Sevo, M. (1997). Keeping the faith: Jewish traditions in pregnancy and childbirth. Lifelines, 46-49.

Doctor search. (2010).The College of Physicians and Surgeons of Ontario. Retrieved on August 7, 2010 http://cpso.on.ca/docsearch/results.aspx

Fatwa 82093: Female being examined by a male doctor. (1999). The Fatwa Center at Islamweb. Retrieved March 31, 2010 from http://www.islamweb.net/ver2/fatwa/ ShowFatwa.php?lang=E\&Id=82093\&Option=Fatwald

Fenwick, J., Hauck, Y., Downie, J., \& Butt, J. (2005). The childbirth expectations of selfselected cohort of Western Australian women. Midwifery: An International Journal, 21(1), 23-35. 
Germain, C. B., \& Bloom, M. (1999). Human behaviour in the social environment. New York: Columbia University Press.

Guruge, S. \& Khanlou, N. (2004). Intersectionalities of Influence: Researching the Health of Immigrant and Refugee Women. Canadian Journal of Nursing Research, $36(3), 32-47$.

Gustafson, D.L. (2008). Are sensitivity and tolerance enough?: Comparing two theoretical approaches to caring for newcomer women with mental health problems. In S. Guruge \& E. Collins (Eds.). (2008). Working with immigrant women: Issues and strategies for mental health professionals (pp.39-63). Toronto, ON: Centre for Addiction and Mental Health (CAMH).

Hutchinson, M.K., \& Baqi-Aziz, M. (1994). Nursing care of the childbearing Muslim family. Journal of Obstetric, Gynecological, and Neonatal Nursing, 23(9), 767-771. Immigration in Canada: A portrait of the foreign-born population, 2006 Census: Portraits of major metropolitan centres. (2006). Statistics Canada. Retrieved on August 24, 2010 from http://www12.statcan.gc.ca/census-recensement/2006/as-sa/97-557/p24eng.cfm

Kabakian-Kasholian, T., Campbell, O., Shediac-Rizakallah, M., \& Ghorayeb, F. (2000). Women's experiences of maternity care: satisfaction or passivity? Social Science \& Medicine, 51, 103-113.

Kerssens, J.J., Bensing, J.M., \& Andela, M.G. (1997). Social Science and Medicine, $44(10), 1531-1540$.

Khalaf, I., \& Callister, L. (1997). Cultural meanings of childbirth: Muslim women living Jordan. Journal of Holistic Nursing, 15, 373-388. 
Khan, M.M. (1996). The Translation of the Meanings of Summarized Sahih-al-

Bukhari:Riyadh: Maktaba Dar-us-Salaam.

Khanlou, N. (2003). Mental health promotion education in multicultural settings. Nurse Education Today, 23(2), 96-103.

Leap, N. (2009, January). Woman-centred or women-centred care: Does it matter? British Journal of Midwifery,17, 12-16.

Lawrence, P. \& Rozmus, C. (2001). Culturally sensitive care for the Muslim patient. Journal of Transcultural Nursing, 12(3), 228-233.

Lurie, N., Margolis, K.L., McGovern, P.G., Mink, P.J., \& Slater, J.S. (1997). Journal of General Internal Medicine, 12, 34-43.

Maimbolwa, M., Yamba, B., Diwan, V., \& Ranjho-Arvidson, A.B. (2003). Cultural childbirth practices and beliefs in Zambia. Journal of Advanced Nursing, 43(3), 263-274.

More women choosing midwives. (2006). Clinical Rounds. Nursing, 36(8), 35. Retrieved on November 28, 2009. http://www.nursingcenter.com/pdf.asp?AID=658496.

Reitmanova S., \& Gustafson D. (2008). “They can't understand it”: Maternity health and care needs of immigrant Muslim women in St. John's, Newfoundland. Maternal and Child Health Journal, 12, 101-111.

Roberts, K. (2002). Providing culturally sensitive care to the childbearing Islamic family. Advances in Neonatal Care, 2, 222-228.

Shorten, A. (1995). Female circumcision: understanding special needs. Holistic Nursing Practice, 9(2), 66-73. 
Simkin, P. (1999). Just another day in a woman's life? Women's long-term perceptions of their first birth experience. Part I. Birth Journal, 18(4), 203-210.

Sperstad, R. A., \& Werner, J.S. (2005). Coming to the cultural "in between": Nursing insights from a Hmong birth case study. Journal of Obstetric, Gynecological, and Neonatal Nursing, 34(6), 682-688.

Spivak, G. C. (1988). Can the subaltern speak? (pp. 271-313). In C. Nelson, \& L. Grossberg. (1988). Marxism and the interpretation of culture. Urbana, Chicago: University of Illinois.

Streubert, H.J., \& Carpenter, D.R. (1999). Qualitative research in nursing: Advancing the humanistic imperative. (2nd ed.). Philadelphia: Lippincott.

Tesch, R. (1990). Qualitative research: Analysis types and software tools. New York: Falmer. pp. 140-145.

The Australian College of Midwives Philosophy Statement for Midwifery. (2010). The Australian College of Midwives. Retrieved August 7, 2010 from http://www.nswmidwives.com.au/links/AustralianCollegeofMidwives/tabid/134/ Default.aspx

The Maternity Experiences Survey. (2006). Statistics Canada. Retrieved March 7, 2010 from http://www.statcan.gc.ca/daily-quotidien/071127/dq071127d-eng.htm Van Manen, M. (1990). Researching lived experience: Human science for an action sensitive pedagogy. NY: State University of New York Press.

Waller, M.A. (2001). Resilience in ecosystemic context: Evolution of the concept. American Journal of Orthopsychiatry, 71(3), 290-297. 
Walsh, D. (1999). An ethnographic study of women's experience of partnership caseload midwifery practice: the professional as a friend. Midwifery. 15(3), 165-176.

What is a midwife? (2010). Association of Ontario Midwives. Retrieved August 19, 2010 from http://www.aom.on.ca/Midwifery_Care/What is_a_Midwife.aspx

Yanikkerem, E., Ozdemir, M., Bingol, H., Tatar, A., \& Karadeniz, G. (2009,October). Women's attitudes and expectations regarding gynaecological examination. Midwifery: An international Journal, 25(5),500-508.

Yosef, A.R.O. (2008). Health beliefs, practice, and priorities for health care of Arab Muslims in the United States. Journal of Transcultural Health, 19(3), 284-291.

Young, D. (2002). Nursing and traditional birth practices: Exploring the need for cultural sensitivity. 16-20. Retrieved November 18, 2009 from www.nursing.aust.edu.au 